\title{
Concentration and self-censorship in commercial media*
}

\author{
Fabrizio Germano \\ Universitat Pompeu Fabra and Barcelona GSE \\ fabrizio.germano@upf.edu \\ Martin Meier \\ Institut für Höhere Studien, Vienna \\ martin.meier@ihs.ac.at
}

December 2010

\begin{abstract}
Within a simple model of non-localized, Hotelling-type competition among arbitrary numbers of media outlets we characterize quality and content of media under different ownership structures. Assuming advertising-sponsored, profit-maximizing outlets, we show that (i) topics sensitive to advertisers can be underreported (self-censored) by all outlets in the market, (ii) self-censorship increases with the concentration of ownership, (iii) adding outlets, while keeping the number of owners fixed, may even increase self-censorship; the latter result relies on consumers' most preferred outlets being potentially owned by the same media companies. We argue that externalities resulting from self-censorship could be empirically large.

Keywords: Media economics; media consolidation; media markets; advertising and commercial media bias. JEL Classification: L13; L82.
\end{abstract}

${ }^{*}$ We are indebted to Matthew Ellman and Joel Sobel for constructive remarks at initial stages of the project and also thank Ramón Caminal, Dries De Smet, Daniel Diermeier, Georgy Egorov, Tim Feddersen, David Genesove, Matthew Gentzkow, Igal Hendel, Bob McChesney, Massimo Motta, Marco Ottaviani, Maria Petrova, Alvaro Sandroni, Mark Satterthwaite, Dan Schiller, Jesse Shapiro, Joel Shapiro, Asher Wolinsky, as well as seminar participants at various seminars and conferences. Financial support from the Spanish Ministry of Science and Technology (Grant SEJ2007-64340), from the Spanish Ministry of Education (in form of a Madariaga Fellowship), from the Fundación Ramón Areces, as well as from the Barcelona GSE Research Network and the Generalitat de Catalunya is gratefully acknowledged. Germano also thanks the CMS-EMS center at Kellogg, Northwestern University, for their generous support and hospitality. 
"The proposal of any new law or regulation of commerce which comes from this order [the profit earners], ought always to be listened to with great precaution, and ought never to be adopted till after having been long and carefully examined, not only with the most scrupulous, but with the most suspicious attention. It comes from an order of men, whose interest is never exactly the same with that of the public, who have generally an interest to deceive and even oppress the public, and who accordingly have upon many occasions, both deceived and oppressed it."

$$
\text { Adam Smith - (I.11.264) The Wealth of Nations }
$$

"The British press is extremely centralised, and most of it is owned by wealthy men who have every motive to be dishonest on certain important topics. But the same kind of veiled censorship also operates in books and periodicals, as well as in plays, films and radio. At any given moment there is an orthodoxy, a body of ideas which it is assumed that all right-thinking people will accept without question." [...] "Anyone who challenges the prevailing orthodoxy finds himself silenced with surprising effectiveness."

George Orwell - proposed preface to Animal Farm

\section{Introduction}

A free, diverse, and independent press has been at the heart of American democracy since the first days of the American Republic. It was clear from the very beginning that this was the cornerstone upon which to build democratic government. In a famous letter to Edward Carrington of January 16, 1787, Thomas Jefferson wrote

"were it left to me to decide whether we should have a government without newspapers, or newspapers without a government, I should not hesitate a moment to prefer the latter. But I should mean that every man should receive those newspapers and be capable of reading them."

It now appears that this very foundation of American democracy is again failing. ${ }^{1}$ Americans are often systematically misinformed about important

\footnotetext{
${ }^{1}$ At the beginning of the twentieth century, commercial influence had become so embedded in US newspapers that each of the three 1912 presidential candidates (Roosevelt,
} 
policy issues such as health care reform, global climate change, the 2003 war in Iraq, the housing and financial crisis of 2008. At the same time, Polls conducted by the Pew center consistently and increasingly show Americans unsatisfied with the quality of their news coverage. ${ }^{2}$

To make matters worse, developments in technology, most notably the internet, are changing the way media are being consumed, and are so far diverting money away from the newsrooms, thus accelerating what now seems to be a crisis in journalism especially in the US (e.g., the Pew Annual Report on the State of the News Media, 2009, 2010, McChesney and Nichols, 2010, Schiffrin, 2010). While the reasons for the crisis are multifaceted and go back several decades, our paper focuses on one particular aspect, commercial media bias, which we believe captures a key fault in American journalism (notice that US media are almost entirely privately owned), but also one we believe may be further significantly exacerbated by the current crisis and possible policy reactions to it.

To address the problem of commercial media bias, we develop an industrial organization model of news markets, where advertising-funded, profitmaximizing and non-ideological media firms decide how accurately and intensively to cover different topics, while consumers are assumed to have a preference for accurate and unbiased information. Our focus is on issues that are sensitive to advertisers (and/or other sponsors).

We show that (i) concentrated media markets can result in self-censorship or severe bias on sensitive topics by all outlets acting optimally in a noncooperative equilibrium; (ii) increasing concentration can deepen the bias;

Tafts, and Wilson) denounced the press's pro-business bias during their campaigns; in the same year Congress passed the Newspaper Publicity Act that required newspapers to list their owners and editors and to clearly demarcate paid advertising from news in order to qualify for postal subsidies (McChesney, 2004, Ch. 2); in 1922 the American Society of Newspaper Editors (ASNE) was finally launched and immediately issued a professional code of ethics for journalists intended to further institute a separation between editorial content and business interests.

${ }^{2}$ E.g, Pew reports of $8 / 6 / 2009$ and $3 / 23 / 2010$ find over $70 \%$ of respondents saying news organizations did either a poor $(>40 \%)$ or a fair job $(>30 \%)$ at explaining proposed health care plans. Also, a Pew report of 9/13/2009 finds a steadily decreasing public's rating of accuracy of news stories (with the number of respondents finding "stories often inaccurate" increasing from $34 \%$ to $63 \%$ between 1985 and 2009). Finally, a Pew report of 5/23/2004 finds similar trends among journalists. 
(iii) increasing the number of subsidiary outlets owned by media companies, keeping the number of owners fixed, can also contribute to deepening the bias, depending among other things on the degree of capture of the audience by individual media firms.

The channel through which bias occurs in our model is the dependence of media on advertising revenues that in turn, indirectly, via the sales of the advertisers' products, depend on the media outlets' coverage. Media outlets internalize advertiser concerns and the effect of their content on sales of advertised products and therefore on advertising budgets. Clearly, issues that are of common concern to advertisers will be more prone to self-censorship. The model also allows for media to charge their audiences.

Media markets are inherently concentrated and to the extent that market profitability cannot accommodate sufficiently many independently owned media firms, absent alternative funding possibilities, the ensuing concentration levels may unavoidably lead to biased coverage. Therefore, in spite of the current difficulties experienced by newspapers and other news firms in the US and elsewhere, our results recommend caution when considering media mergers as a possible solution. We do not exclude public funding of media as a possible, and for the time being necessary, way out. ${ }^{3}$

To illustrate our notion of bias and to give a sense of the possible externalities involved, we consider two examples: the coverage of tobacco related health hazards in US media since the 1940's and the more recent coverage of anthropogenic climate change. Both involve (or involved) industries with substantial advertising budgets. ${ }^{4}$

\footnotetext{
${ }^{3}$ Clearly, government bias is a reason to be skeptical, but as e.g., McChesney and Nichols (2010) stress, government funding of media was, by today's standards, not only substantial in the early days of the American Republic, but it was also understood to be vital to the democratic process (the amount spent in 1840 as percentage of GDP, largely for postal subsidies, would correspond to some $\$ 30$ billion today). They also emphasize how many or most of the more advanced nations in the world (by many accounts like the UN's human development index, environmental sustainability, and other political or press freedom indices) have high rates of public funding of their media. Other important possibilities inculde setting up endowments for media. See also Schiffrin (2010).

${ }^{4}$ Tobacco companies such as Brown $\mathcal{E}$ Williamson (part of British American Tobacco) or Philip Morris (previously part of Altria Group) as well as for instance car manufacturers such as General Motors or Ford have consistently been top advertisers in the US at different points in time (e.g., Baker, 1994, and Advertising Age, 2007).
} 
For decades, despite hundreds of thousands of deaths a year, serious statistical and medical evidence about the health hazards of smoking were kept away from mainstream commercial media (e.g., Baker, 1994, and Bagdikian, 2004, have chronologies, Chaloupka and Warner, 2000, discusses statistical evidence on the impact of advertising on the (non-)coverage of tobacco related health hazards). Bagdikian (2004, pp. 250-252) summarizes

"In 1980 [...] there were still more stories in the daily press about the causes of influenza, polio, and tuberculosis than about the cause of one in every seven deaths in the United States," so that "[a]s late as fourteen years after the Surgeon General cited serious health risks from smoking, and seven years after the Surgeon General declared that even second-hand smoking may cause lung cancer, 64 million Americans, obviously already addicted, smoked an average of 26 cigarettes a day."

Baker (1994, p. 51) adds that in the same period surveys indicated that "half the general and two-thirds the smoking population [did] not think smoking made 'a great deal of difference' in life expectancy."

This paper claims that, alongside advertising, concentration in media markets plays an important role in aggravating the bias. By focusing on industrial organization aspects of the media markets our framework allows to quantify the role of the media ownership structure on the degree of self-censorship.

Our second example concerns the coverage of anthropogenic climate change. In a comprehensive study of the scientific literature, Oreskes (2004) finds that of all the 928 peer-reviewed papers published between 1993-2003 none (0\%) disagree with the "scientific consensus position" that "most of the observed global warming over the last 50 years is due to the greenhouse gas concentration." At the same time, in a study of the US newspaper coverage, Boykoff and Boykoff (2004) find that over half (53\%) of a random sample of articles published in quality US national newspapers ${ }^{6}$ between 1988-2002, give

\footnotetext{
${ }^{5}$ In 2009, according to the Center for Disease Control and Prevention of the US Department of Health and Human Services, cigarette smoking remained the leading preventable cause of death in the US, accounting for approximately 1 of every 5 deaths $(=443,000$ people; including 49.400 deaths from second hand smoking) per year.

${ }^{6}$ New York Times, Washington Post, Los Angeles Times, and Wall Street Journal.
} 
equal attention to the scientific consensus position on one side and to the industry-supported view on the other that "natural fluctuations suffice to explain global warming." Consistent with our model, Boykoff (2008) finds even more bias in the US TV news ( $70 \%$ of randomly selected news segments ${ }^{7}$ giving the "balanced" view) which are both more heavily funded by advertising and also more concentrated than the US national print media; also, in a similar study of the Indian news coverage, Billett (2010) finds dramatically less bias among quality Indian national newspapers in English language, ${ }^{8}$ where there is almost a complete endorsement of the scientific consensus position ( $98 \%$ of randomly selected articles). Notice that the Indian newspaper market is substantially more competitive than the US one. This takes us to the next point: the role of concentrated media markets.

A further motivation for our analysis is the ongoing media policy debate in the United States, which especially following the Telecommunications Act of 1996, has seen a significant wave of consolidation in US media industries. Moreover, in a media landscape that is arguably exceedingly concentrated already, ${ }^{9}$ the Federal Communications Commission has attempted twice (in 2003 and 2007) to further loosen ownership rules (McChesney, 2004, contains an extensive discussion of the 2003 attempt).

While empirical evidence on the effects of concentration on commercial bias or quality of coverage is admittedly meager, a PEJ study of local television news over a five year period summarizes that "overall the data strongly suggest [..] heavy concentration of ownership in local television by a few large corporations will erode the quality of news Americans receive." 10 Moreover,

\footnotetext{
${ }^{7}$ Taken from $A B C$ World News Tonight, CBS Evening News, NBC Nightly News and also from three $C N N$ programs.

${ }^{8}$ Times of India, Hindu, Hindustan Times, and Indian Express.

${ }^{9}$ According to Bagdikian (2004) five media conglomerates (Time Warner, Disney, News Corporation, Viacom, and Bertelsman) produce more than half of all of US mass media consumption; a number he put around fifty in the early 1980's, claiming excessive concentration even then. Compaine and Gomery (2000) and Noam (2009) contain important qualifications and updates of these figures; see also Baker (2007) on the notion of "relevant" market; McChesney (2004) speaks of three-tiered media markets.

${ }^{10}$ PEJ study of $4 / 23 / 2003$, "Does Ownership Matter in Local Television News?" Lacy and Blanchard (2003) find indirect evidence that competition increases quality of news reporting. Ho and Quinn (2009) find mixed evidence of mergers on reporting diversity of editorials on nonunanimous Supreme Court decisions in 25 top newspapers from 1988-2003.
} 
trends emerging from Pew reports mentioned above on increasing dissatisfaction among consumers and journalists documented since the mid 1980's are consistent with our results given the simultaneous rise in general media concentration. ${ }^{11}$ Evidence on the result that increasing the number of subsidiary outlets owned, while keeping the number of owners fixed, can adversely affect accuracy, is even weaker. Though, possibly the most pronounced finding of the above mentioned PEJ study of 4/23/2003 is that "smaller [TV] station groups tended to produce higher quality newscasts than stations owned by larger companies by a significant margin." Clearly more empirical evidence is needed to link some of these trends to the more specific variables emerging from our analysis.

At the center of our model are media outlets' advertising revenues and a generic information variable $(\mathbf{x})$ measuring the amount of information provided by the outlets on "sensitive" topics. We make the following key assumptions: (A1) Advertisers spend a fixed fraction $(\eta>0)$ of their sales on ads; (A2) advertisers advertise with all outlets in proportion to their audience shares $\left(s_{i} \geq 0\right)$; (A3) final sales of advertised products can be written as $C(\mathbf{x})=\varphi(x) C_{0}$, where $\varphi(\cdot)$ is a decreasing function of the sensitive information variable $\mathbf{x}$, and $C_{0}$ is an exogenously given level of base consumption of advertised products. Assumptions (A1)-(A3) combined imply that media outlet $i$ 's advertising revenues can be written as $s_{i} \eta C(\mathbf{x})$, where $C(\cdot)$ is decreasing in $\mathbf{x}$.

The idea of (A3) is that repeated exposure to sensitive information may put off consumers from buying certain products or may make them disposed towards policies that eventually decrease final expenditures on advertised products and hence decrease advertising revenues of media outlets. (A1) and (A2) are essentially approximations. Schmalensee (1972) shows that (A1) can be optimal in many cases and also provides empirical support; Baghestani

\footnotetext{
${ }^{11}$ E.g., a Pew report of 9/13/2009 finds that the number of respondents thinking "[n]ews organizations are influenced by powerful people/organizations" increased from $53 \%$ to $74 \%$ between 1985 and 2009; simiarly, a Pew report of 5/23/2004 finds, among other things, that journalists "fear more than ever that the economic behavior of their companies is eroding the quality of journalism. In particular, they think business pressures are making the news they produce thinner and shallower. And they report more cases of advertisers and owners breaching the independence of the newsroom."
} 
(1991), Jung and Seldon (1995), and Elliott (2001) contain further empirical evidence; (A2) implicitly assumes advertisers value consumers equally, and essentially abstracts from issues of targeting, whereby advertisers strategically target audiences of particular interest to them. ${ }^{12}$

In particular, we do not model the "direct" (punishment) effect advertisers may have on media outlets if they (or advertising agencies representing them) can withdraw their ads in response to excessively critical coverage. This is modeled in detail in Ellman and Germano (2009, Section 4) and shown to lead to underreporting on sensitive topics also by competing outlets. Here instead we focus on the "indirect" channel that occurs through reduced sales of advertised products. While we believe both effects are empirically relevant, it is with the indirect effect that concentration plays a more important role. Because of the public good nature of the information variable $\mathbf{x}$, the more concentrated the media markets, the more media firms internalize the (indirect) effect of their reporting on sales of advertised products and hence on their own advertising revenues, and the more they are willing to underreport sensitive information.

A further interpretation of the variable $\mathbf{x}$ - probably more pertinent to TV outlets - is the amount of "critical" programming in the sense of the inverse of "dumbed down" content. The latter may improve the effectiveness of advertising on the reception and eventual consumption of advertised products (see, e.g., Ellman and Germano, 2009, Section 1.5). Baker (1994, 2007), Bagdikian (2004), McChesney (2004), and Hamilton (2004) present evidence suggesting an increase in "dumbed down" content in the US over the last decades, which is consistent with our results given the simultaneous increase in media concentration. Clearly, a rigorous and empirical analysis of the

\footnotetext{
${ }^{12}$ Allowing for targeting may actually strengthen our results in the following sense. Assume advertisers concentrate their advertising budget on a subset of outlets. Then self-censorship on sensitive topics would be even more likely on those outlets, while it may well be absent on the other ones. Average awareness might be higher than without targeting. However, to the extent that (negtive) externalities from not reporting on these sensitive topics originate from consumption of the products, since it is mainly the targeted audience consuming the advertised products, self-censorship when allowing for targeting being higher for the targeted audience, could mean higher consumption and so even lower overall welfare through the the externalities.
} 
relationship needs to be carried out. ${ }^{13}$

Related literature. Meanwhile there is a large and growing economics literature on the role of competition on quality of media provision. In an early paper, Steiner (1952) shows how a monopoly firm owning multiple media outlets can provide coverage that is more differentiatiated and so closer to the social optimum than if the outlets were separately owned. This has been an important argument favoring consolidation.

More recently, Gentzkow and Shapiro (2008a) survey the literature on media bias and competition and distinguish between demand and supply driven bias. Competition is generally seen as being relatively ineffective in disciplining bias that is driven by the audience's preferences, (e.g., Mullainathan and Shleifer, 2005), whereas, it can play an important role in mitigating bias driven on the supply side (e.g., Besley and Prat, 2006). Most of the economics literature focuses on political influence in the media. An important class of models relating to commercial influence are the targeting models, where programming is tailored to viewers most valued by advertisers. This leads to corresponding biases in programming (e.g., cultural bias as in George and Waldfogel, 2003, or political bias as in Strömberg, 2004; see also Hamilton, 2004) which are also ultimately driven by the preferences of the audience (who are targeted) and so again competition need not help to reduce this kind of bias. Gal-Or et al. (2010) combines both audience and advertiser driven biases and shows some important qualifications of the polarizing effects of competition derived in Mullainathan and Shleifer (2005).

Ellman and Germano (2009) study advertiser influence and explicitly model advertisers and consumers, besides the media outlets. In particular, they allow advertisers to choose with whom to advertise and whether to withdraw their ads from individual outlets upon observing too critical cover-

\footnotetext{
${ }^{13}$ Another related aspect concerns the quality of educational, social, or "public interest" content of commercial media. Downs (1957) famously argued that this type of content would be underprovided by commercial media, see also Hamilton (2004); Putnam (2000) attributes a substantial part of the civil disengagement he documents in the United States to TV and other electronic media. Testing commercial media's performance in this respect seems worthwhile. Prat and Strömberg (2006) and Gentzkow and Shapiro (2008b) present some evidence on this.
} 
age (direct effect). Competition is generally beneficial in reducing advertiser bias, but it need not always help. Blasco et al. (2010) study an interesting model where advertisers compete among each other in order to get favored coverage from competing media firms.

Armstrong and Weeds (2007) also use a Hotelling-type model to evaluate the role of advertising, pay TV, and public broadcasting on the quality of programming. Their focus with advertising concerns the funding of programming and the disutility from having to watch ads (see also e.g., Anderson and Coate, 2005). To their analysis our paper adds that advertising may not only cause welfare loss through the disutility of watching ads but also through its effect on content. As is clear from our examples, this can lead to substantial externalities that should be taken into account when computing the "efficient" amount of advertising. More particularly it suggests caution when recommending abandoning public broadcasting.

Finally, there are several empirical papers estimating the effect of advertising on media coverage. Reuter and Zitzewitz (2006) provides empirical evidence of bias favoring mutual funds advertising in financial publications. Gambaro and Puglisi (2009), Rinallo and Basuroy (2009), and De Smet and Vanormelingen (2010) present similar evidence for broader sets of advertisers. Wilbur (2008) uses a two-sided market framework to show that TV network program choices are influenced more by advertiser than by viewer preferences. These papers also generally show how larger advertising budgets lead to more bias, but they do not address the role of concentration or consolidation in the media.

The paper is organized as follows. Section 2 sets out the basic model used throughout the paper, Section 3 contains all the main results while Section 4 contains a brief welfare analysis. The robustness and some extensions of the results are explored in Section 5, and finally Section 6 concludes. Most proofs are contained in the Appendix. 


\section{The Framework}

In order to capture multiple outlets all in competition with each other, we work with Chen and Riordan's (2007) generalization of Hotelling's model that in our aplication allows for $n$ media outlets to compete for audience (and indirectly advertisers) in a non-localized fashion.

There are $n(\geq 2)$ commercial media outlets, assumed to maximize profits derived from advertising and payments from the audience minus costs of producing the programming, that are located at the $n$ endpoints of a starshaped network with $n$ spokes (one for each outlet). Each endpoint has a distance $\frac{1}{2}$ to the center of the network so that any two endpoints (and therefore any two outlets) have distance 1 from each other. (In particular, there is no outlet located at the center.)

There is a mass one of consumers uniformly distributed along the $n$ spokes of the network. As in Chen and Riordan (2007), consumers receive a positive valuation $v>0$ and therefore have a preference for only two of the $n$ outlets, namely, the outlet corresponding to the spoke the consumer is located on and another one chosen at random with uniform probability from all the remaining $n-1$ outlets. ${ }^{14}$ This guarantees continuity and significantly simplifies the analysis.

When having to "travel" from their location to one of the two preferred outlets, consumers incur transportation costs measured at a fixed rate $t>0$ per unit distance. Therefore, since any two points on the network have distance at most 1 from each other, transportation costs are between 0 and $t$, depending on the location of the consumer: A consumer located at the endpoint of a spoke incurs a transportation cost of 0 for consuming the outlet located at the same endpoint, and incurs a cost of $t$ for consuming the other outlet instead; otherwise, consumers incur transportation costs less than $\frac{t}{2}$ to consume the outlet at the endpoint of the spoke on which they are located, and greater than $\frac{t}{2}$ to consume the other one instead; clearly, the closer the

\footnotetext{
${ }^{14}$ Chen and Riordan's (2007) original model allows for the "uncovered" case with $N(\geq n)$ potential and $n$ actual brands, so that if $N>n$ some consumers may have a preference for just one or zero actually available brands. We study the "covered" case where $N=n$, where each consumer has a preference for exactly two brands.
} 
consumer is located to the center the closer the transportation cost to any outlet will be to $\frac{t}{2}$. Finally, to get a sense of its size, $t$ can be interpreted loosely as representing the maximum that "most faithful" readers are willing to pay to access their most preferred newspaper when forced to read their second-best choice instead.

There is an exogenous degree of horizontal product differentiation between the media outlets so that shares are determined by the equation

$$
s_{i}=\frac{1}{n}+\frac{1}{n(n-1)} \sum_{j \neq i} s_{i j}
$$

where $s_{i j}$ is the share of viewers on $j$ 's spoke that $i$ appropriates from $j$,

$$
s_{i j}=\left\{\begin{array}{cl}
-1 & \text { if } u_{i}-u_{j}<-t \\
\frac{u_{i}-u_{j}}{t} & \text { if }\left|u_{i}-u_{j}\right| \leq t \\
1 & \text { if } u_{i}-u_{j}>t
\end{array}\right.
$$

where $t>0$ is the transportation cost and $u_{i}$ is the utility (gross of transportation costs) offered by outlet $i$.

Specifically, the utility offered by outlet $i$ is defined by

$$
u_{i}=v+\alpha x_{i}+\beta y_{i}-p_{i}
$$

where $v \gg \alpha+\beta$ is the fixed exogenous valuation, which for any consumer is positive for exactly two outlets: the one at the endpoint of the spoke where the consumer is located, and the other preferred outlet; $x_{i} \in[0,1]$ is accuracy or amount of information reported on sensitive topics; $y_{i} \in[0,1]$ is an endogenous measure of quality unrelated to $x_{i} ; p_{i} \geq 0$ is the price charged by outlet $i ; 0 \leq \alpha, \beta \leq t$ are parameters. To get the overall utility of outlet $i$ to a consumer located at some point $\ell$ on the network, one subtracts from $u_{i}$ the transportation cost, obtained as $t$ multiplied with the distance between $\ell$ and the endpoint where outlet $i$ is located.

Assuming $\left|u_{i}-u_{j}\right| \leq t$, which we assume throughout unless otherwise stated, the share equation reduces to

$$
s_{i}=\frac{1}{n}+\frac{1}{n(n-1) t} \sum_{j \neq i}\left(u_{i}-u_{j}\right)=\frac{1}{n}+\frac{u_{i}-u}{n t},
$$


where $u=\frac{1}{n-1} \sum_{j \neq i} u_{j}$ is averaged over outlets other than $i$. Unlike the Salop model, where a given firm can essentially only compete with its two neighbors, here all outlets are in direct competition with each other. Any two pair of outlets compete over a share of $\frac{2}{n(n-1)}$ consumers. Their strategic variables are $x_{i}, y_{i}$, and $p_{i}$.

Expenditure on advertiser products plays a crucial role throughout the paper since it drives advertising revenues of media firms and at the same time makes the link with "sensitive" information $x_{i}$. We assume aggregate expenditure takes the reduced form

$$
C(\mathbf{x})=\left(1-\psi \sum_{i=1}^{n} s_{i} x_{i}\right) \cdot C_{0},
$$

where $\mathbf{x}=\left(x_{1}, \ldots, x_{n}\right)$ is the vector of information levels of the different outlets, and $\psi \in[0,1)$ is a constant representing the marginal effect of information on final aggregate expenditure. This assumes that more accurate information (or less bias) captured by a higher value of the $x_{i}$ 's depresses overall expenditure at the rate $\psi{ }^{15}$

Media outlets maximize profits, which for outlet $i$ are given by

$$
\pi_{i}=s_{i} \eta C(\mathbf{x})+s_{i} p_{i}-\frac{\delta}{2} y_{i}^{2},
$$

where $C(\cdot)$ is total expenditure defined in Eq. (4), and $\eta$ and $\delta$ are fixed parameters satisfying $0 \leq \eta<1, \delta>\frac{\eta C_{0}}{n}$. (The assumption on $\delta$ ensures that $y_{i} \in[0,1]$.) The first and second expressions represent revenues from advertising and from the audience respectively; the third expression represents variable costs of producing quality or programming $(y)$. We assume that advertising revenues are a fixed share of total sales of the advertised products weighted by the audience share of the outlet.

The main object of the present paper can be viewed as studying the implications of a positive $\psi$ on the strategic variables of media firms, especially, the effect on the accuracy variable $\mathbf{x}$.

\footnotetext{
${ }^{15}$ More precisely, outlet $i$ increasing $x_{i}$ depresses expenditure by a factor $s_{i} \psi$. This implicitly assumes that information provided by outlet $i$ does not spread to audiences of other outlets. In Section 5 we show that allowing information to spread across other outlets' audiences can lead to even more self-censorship.
} 
Some Interpretations. Generally speaking, the information variable $\mathbf{x}$ represents a variety of news topics that can range from how products are produced to what they may contain and whether they satisfy certain standards or ethical norms, all the way to what health care system should be adopted or whether severe measures should be taken to reduce greenhouse gas emissions. Since the model is static, $\mathbf{x}$ can be thought of as a long-term accuracy of reporting measure for the different outlets, and we assume that it is consistent with the standard norms of professional journalism.

Ellman and Germano (2009, Section 1.5) contain further discussion and interpretation as well as a simple derivation, within a context of Bayesian rational agents, of the fact that accuracy as measured by a variable such as $\mathbf{x}$ (where higher $\mathbf{x}$ means less suppression of bad news) may have a negative impact on expected quantities sold by advertisers. This can be done even if (consistent with journalism standards) media outlets do not falsify information. Essentially, it follows if bad news about advertisers' products can be withheld (without the consumers knowing exactly what is withheld), and so this can shift downwards consumers' posteriors on the value of the products advertised. Anderson and McLaren (2010) contains a related derivation.

Clearly, to the extent that $\mathrm{x}$ represents "bad" news that affects all advertisers, the effect will be larger than if it only affects few advertisers, or it will be larger than if some of the affected advertisers' products can be substituted with other advertised products. We do not in fact exclude that accuracy on topics that negatively affect some advertisers may benefit others (we come back to this in Section 5.1), but the focus throughout is on the case where the net effect on demand is negative, namely of the order $-\psi$.

Also worth pointing out is that consumers are assumed to be aware of the level of $\mathbf{x}$ as media outlets are implicitly assumed to be evaluated over periods of time long enough for readers to make an opinion about the reputation and accuracy of coverage. However, we do not exclude that a low value of $\alpha$ can reflect readers not much aware of sensitive issues (we come back to this question in Section 5.5). When $\mathbf{x}$ concerns public issues, a small value of $\alpha$ may also reflect free-riding on others to be informed, as argued for instance by Downs (1957) and Hamilton (2004). 
Finally, when comparing networks or ownership structures with different numbers of outlets or firms we understand that consumers are redistributed along the netwrok according to an (unmodeled) equilibrium. To emphasize the robustness of our main results, however, in Section 3.5, we consider alternative frameworks, namely, the Salop and the mulinomial logit models, ${ }^{16}$ where the preferences of the consumers (location along the network) remain fixed when changing the ownership structure. Germano (2009) studies the original "uncovered" spokes model of Chen and Riordan (2007).

\section{Self-censorship in commercial media}

Commercial media are assumed to maximize profits as described in Eq. (5). We start our study of commercial media bias with the case of purely advertising funded media, before turning to the more general case where revenues include payments from the audience.

\subsection{Purely advertising funded media}

Here we consider purely advertising funded media so that $p_{i}=0$ by assumption for all outlets $i \in N$. From the symmetric FOC's (see the appendix for a complete derivation) we get

$$
\begin{aligned}
& \frac{\partial \pi}{\partial x_{i}}=\frac{\eta C_{0}}{n t}\left(\alpha(1-\psi x)-\frac{\psi t}{n}\right)=0 \\
& \frac{\partial \pi_{i}}{\partial y_{i}}=\frac{\beta \eta}{n t}(1-\psi x) C_{0}-\delta y=0
\end{aligned}
$$

which, yields $y=\frac{\beta \eta}{\delta n t}(1-\psi x) C_{0}$ for the level of quality, while for the level of sensitive information, solving for unrestricted $x \in \mathbb{R}$, leads to

$$
x(n)=\frac{1}{\psi}-\frac{t}{\alpha n}
$$

as the locus of $(x, n)$ 's such that $\frac{\partial \pi}{\partial x_{i}}=0$ (notice that the SOC's are automatically satisfied here). To take into account the constraint $x \in[0,1]$ we

\footnotetext{
${ }^{16}$ See respectively, Tirole (1988) and Anderson et al. (1992) for expositions.
} 


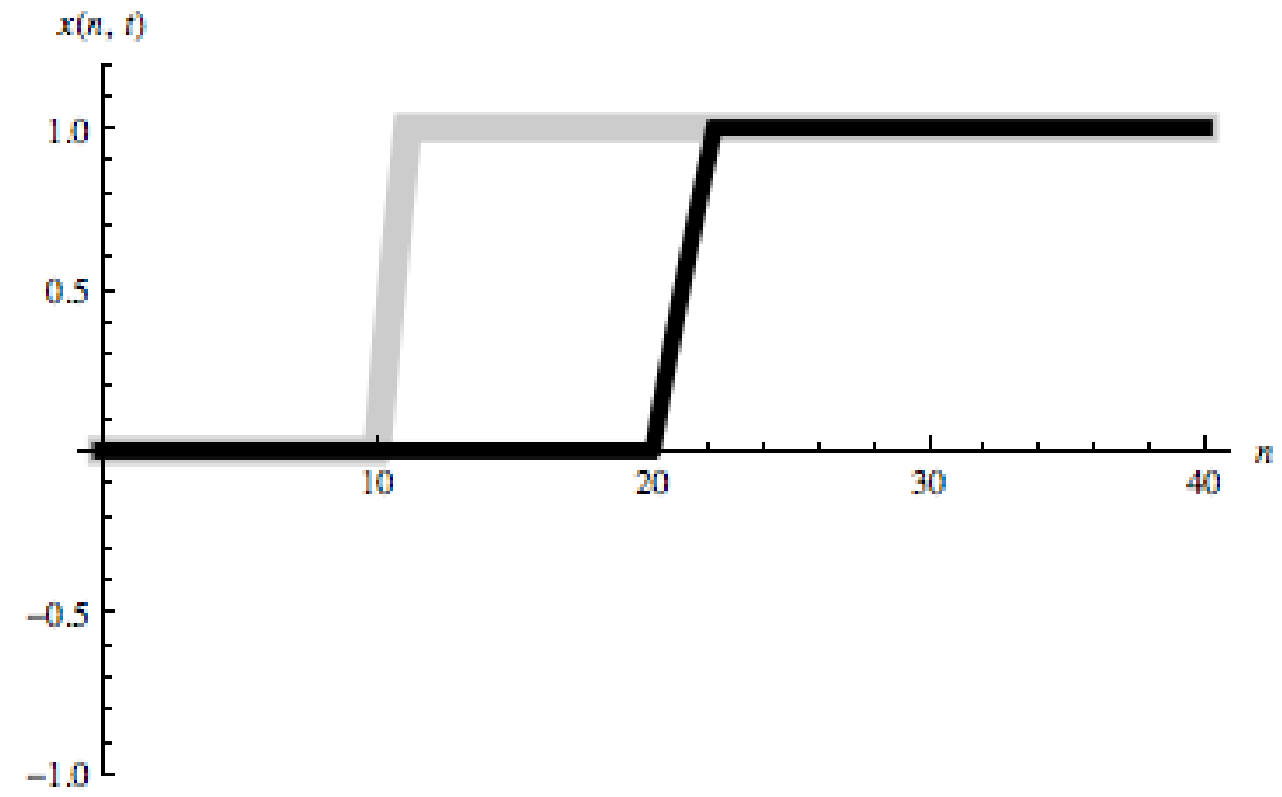

Figure 1: $x(n)$ as a function of $n[t=5$ (grey) or 10 (black); $\alpha=\psi=.1]$

solve $x(n)=0$ and $x(n)=1$, which yields respectively

$$
\bar{n}=\frac{\psi t}{\alpha} \quad \text { and } \quad \overline{\bar{n}}=\frac{\psi t}{\alpha(1-\psi)},
$$

so that, for $n<\bar{n}$, we have $x=0$ as the unique solution; while for $n>\overline{\bar{n}}$, we have $x=1$ as the unique solution; for intermediate values $n \in[\bar{n}, \overline{\bar{n}}]$ we have $x=\frac{1}{\psi}-\frac{t}{\alpha n} \in[0,1]$, overall,

$$
x= \begin{cases}0 & \text { if } n<\bar{n} \\ \frac{1}{\psi}-\frac{t}{\alpha n} & \text { if } \bar{n} \leq n<\overline{\bar{n}} \\ 1 & \text { if } n \geq \overline{\bar{n}}\end{cases}
$$

We can state our first main result.

Proposition 1 In a market with $n$ purely advertising funded media outlets there is a unique symmetric equilibrium with: severe bias $\left(x_{i}=0\right.$ for all $\left.i\right)$ if $n<\bar{n}$; no bias $\left(x_{i}=1\right.$ for all $\left.i\right)$ if $n \geq \overline{\bar{n}}$; intermediate bias $\left(x_{i}=\frac{1}{\psi}-\frac{t}{\alpha n} \in\right.$ $[0,1]$ for all $i)$ in the range $\bar{n} \leq n<\overline{\bar{n}}$, where $\bar{n}=\frac{\psi t}{\alpha}$ and $\overline{\bar{n}}=\frac{\psi t}{\alpha(1-\psi)}$. The level of quality is given by $y_{i}=\frac{\beta \eta C(x)}{\delta n t}$ where $x=x_{i}$ for all $i$. 
Severe media bias (or self-censorship) occurs when too few outlets $(n<\bar{n})$ are active in the market; in equilibrium they all endogeneously choose the lowest possible level of reporting on sensitive topics $(x=0)$. The larger the transportation costs $(t)$ and the marginal effect of information on consumption $(\psi)$, and the smaller the preference for accuracy parameter $(\alpha)$, the more outlets are needed to avoid self-censorship. Two reasons the parameter $\alpha$ can be low are if there is already low awareness of the issues to begin with, or if, as Downs (1957) has argued, viewers free ride on others being informed. On the other hand, when transportation costs are sufficiently small, there is always a unique, fully informative equilibrium. ${ }^{17}$

The result follows essentially from the upward slopedness of the $(x, n)$ locus, Eq. (6), which says that, as the number of outlets $n$ increases, firms want to raise their level of accuracy $x_{i}$. To see this, let the elasticities $\epsilon_{s_{i}}=\frac{\partial s_{i}}{\partial x_{i}} \frac{x_{i}}{s_{i}}$ and $\left|\epsilon_{C}\right|=\left|\frac{\partial C}{\partial x_{i}} \frac{x_{i}}{C}\right|$ measure what we call respectively the competitive and the externality effect of rasing $x_{i}$. As $n$ increases firms raise accuracy since they internalize less the effect on sales and hence on advertising revenues. This in turn follows from the fact that (at the unrestricted symmetric solution $x$ ) the competitive effect is (weakly) increasing in $n$ while the externality effect is strictly decreasing with $n$. These relationships hold fairly generally and both reinforce a positive relation between $n$ and $x .^{18}$

The additional fact that the $(x, n)$ locus lies below zero when $n<\bar{n}$ has to do with the linear form of the utility functions $u_{i}$. Clearly, if preferences between $x_{i}$ and $y_{i}$ were say of the Cobb-Douglas type, then setting either $x_{i}$ or $y_{i}$ equal to zero would be very costly for the media outlets. In that case the locus would be above zero for low values of $n$. However, it is unclear

\footnotetext{
${ }^{17}$ Firms have an interest to have $t$ large as it increases profits through either allowing them to decrease $x, y$ or as we will see also to raise $p$. In this sense, one may view certain choices of programming or programming styles as trying to increase $t$.

${ }^{18}$ More specifically, they can be shown to imply that the slope of the $(x, n)$ locus computed from the FOC condition for $x_{i}, \frac{\partial \pi_{i}}{\partial x_{i}}=0$, generally satisfies

$$
\frac{d x_{i}}{d n}=-\frac{\partial\left[\epsilon_{s_{i}} / \epsilon_{C}\right] / \partial n}{\partial\left[\epsilon_{s_{i}} / \epsilon_{C}\right] / \partial x_{i}}=-\frac{\left[\frac{\partial \epsilon_{s_{i}}}{\partial n} \epsilon_{C}-\epsilon_{s_{i}} \frac{\partial \epsilon_{C}}{\partial n}\right] /\left(\epsilon_{C}\right)^{2}}{\left[\frac{\partial s_{i}}{\partial x_{i}} / \frac{\partial C}{\partial x_{i}}\right] \frac{\partial\left[C / s_{i}\right]}{\partial x_{i}}}>0,
$$

provided the second order derivatives $\frac{\partial^{2} s_{i}}{\partial x_{i}^{2}}$ and $\frac{\partial^{2} C}{\partial x_{i}^{2}}$ are vanishingly small.
} 
whether this would be a more realistic way of capturing preferences.

\subsection{Audience and advertising funded media}

We now consider the more general case described at the beginning of the section and solve for symmetric equilibria where all media outlets choose simultaneously accuracy on the sensitive topic $x_{i}$, quality $y_{i}$, and prices charged $p_{i}$. In particular, we now allow media outlets to be funded by the audience through viewer or reader fees $p_{i}$.

Rewriting the symmetric FOC's for the general case we have

$$
\begin{aligned}
\frac{\partial \pi_{i}}{\partial x_{i}} & =\frac{\alpha \eta C_{0}}{n t}\left((1-\psi x)-\frac{\psi t}{\alpha n}\right)+\frac{\alpha}{n t} p=0 \\
\frac{\partial \pi_{i}}{\partial y_{i}} & =\frac{\beta \eta C_{0}}{n t}(1-\psi x)+\frac{\beta}{n t} p-\delta y=0 \\
\frac{\partial \pi_{i}}{\partial p_{i}} & =-\frac{\eta C_{0}}{n t}(1-\psi x)+\frac{1}{n}-\frac{1}{n t} p=0 .
\end{aligned}
$$

Solving for $(x, y, p)$ yields

$$
p=[t-\eta C(x)]^{+}, \quad y=\frac{\beta \eta C(x)}{\delta n t}+\frac{\beta[t-\eta C(x)]^{+}}{n t}
$$

where $x$ is again given by Eq. (7) and the values of $\bar{n}$ and $\overline{\bar{n}}$ are now

$$
\bar{n}=\min \left\{\frac{\psi t}{\alpha}, \frac{\psi \eta C_{0}}{\alpha}\right\}, \quad \overline{\bar{n}}=\min \left\{\frac{\psi t}{\alpha(1-\psi)}, \frac{\psi \eta C_{0}}{\alpha}\right\},
$$

so that if $t \geq \eta C_{0}$ then $x$ is either 0 or 1 . We can state the following.

Proposition 2 In a market with $n$ advertising and audience funded media outlets the unique symmetric equilibrium has the following properties:

(a) If potential advertising revenues are large relative to transportation costs $\left(t<\eta C_{0}\right),{ }^{19}$ then media outlets will choose not to charge their audience $\left(p_{i}=0\right.$ for all $\left.i\right)$, and will be exclusively advertising funded. The equilibrium levels of bias $(x)$ and quality $(y)$ coincide with the ones of Proposition 1.

\footnotetext{
${ }^{19}$ Essentially the condition states that transportation costs $(t)$ are no greater than the maximal total advertising revenue per capita $\left(\eta C_{0}\right)$ achieved when $x_{i}=0$ for all $i$.
} 
(b) If potential advertising revenues are small relative to transportation costs $\left(t \geq \eta C_{0}\right)$, then media outlets will charge positive prices $\left(p_{i}=t-\right.$ $\eta C(x)$ ), and there will be: severe bias $\left(x_{i}=0\right.$ for all $\left.i\right)$ if $n<\hat{n}$ and no bias

$\left(x_{i}=1\right.$ for all $\left.i\right)$ if $n \geq \hat{n}$, where $\hat{n}=\frac{\psi \eta C_{0}}{\alpha}$. The level of quality is given by $y_{i}=\frac{\beta}{n}+\frac{\beta \eta(1-\delta) C(x)}{\delta n t}$, where $x_{i}=x$ for all $i$.

One can think of the two polar cases considered in the statement, namely small or large transportation costs relative to potential advertising revenues, for instance as reflecting respectively the cases of free to air television (for (a)) and print newspapers (for (b)).

Prices are charged only to the extent that advertising revenues are not sufficient to cover the transportation cost $\left(p=[t-\eta C(x)]^{+}\right)$. In case (a) outlets have sufficient advertising revenues that they voluntarily choose not to charge the audience. The number of media outlets necessary to avoid severe bias is smaller in (b) and increases with the amount of revenues obtained from advertising $\left(\hat{n}=\frac{\psi \eta C_{0}}{\alpha}\right)$.

\subsection{Purely audience funded media}

If we consider the other limit case of media funded exclusively by the audience who faces a price $p_{i}$ for accessing outlet $i$, then by setting $\eta=0$ in the previous analysis, we immediately get the following statement.

Proposition 3 In a market with $n$ purely audience funded media outlets there is a unique symmetric equilibrium with no bias $\left(x_{i}=1\right.$ for all $\left.i\right)$ at positive prices $\left(p_{i}=t\right.$ for all $\left.i\right)$ for any $n \geq 2$. The quality provided is $y=\frac{\beta}{\delta n}$.

Since there is no funding by advertisers, the audience pays the full fee $t$ (instead of $[t-\eta C(x)]^{+}$). This guarantees (essentially by assumption) that there will be no bias (on advertiser-sensitive topics) and that the efficient quantities for both $x$ and $y$ are implemented; though this may not necessarily be preferred by consumers or producers. 


\subsection{Multiple ownership}

In practice a given media firm may own several different media outlets which may be in direct competition with each other. ${ }^{20}$ For simplicity, we consider the case where each media firm owns the same number $(\kappa)$ of media outlets, and to begin with also assume they are all purely advertising funded. Let $n$ denote the total number of firms (owners of the outlets), and suppose each firm owns $\kappa \geq 1$ media outlets. Each firm $i$ 's profits are then given by

$$
\pi_{i}=\sum_{\kappa_{i}=1}^{\kappa}\left(s_{\kappa_{i}} \eta C(\mathbf{x})-\frac{\delta}{2} y_{\kappa_{i}}^{2}\right)
$$

where now $\mathbf{x}=\left(x_{1}, x_{2}, \ldots, x_{\kappa n}\right) \in[0,1]^{\kappa n}$. From the FOC's under symmetry $\left(x_{\kappa_{j}}=x\right.$ for all $j$ and $\left.\kappa_{j}\right)$ we have

$$
\frac{\partial \pi}{\partial x_{\kappa_{i}}}=\frac{\eta C_{0}}{\kappa n}\left(\frac{\alpha \kappa(n-1)(1-\psi x)}{(\kappa n-1) t}-\frac{\psi}{n}\right),
$$

which, solving for unrestricted $x \in \mathbb{R}$, yields

$$
x(n, \kappa)=\frac{1}{\psi}-\frac{(\kappa n-1) t}{\alpha \kappa n(n-1)}
$$

as the locus of unrestricted $(x, n)$ 's such that $\frac{\partial \pi_{i}}{\partial x_{k_{i}}}=0$. Taking into account the constraint $x \in[0,1]$ we obtain (again the second order conditions are always satisfied here),

$$
x= \begin{cases}0 & \text { if } n<\bar{n}(\kappa) \\ \frac{1}{\psi}-\frac{(\kappa n-1) t}{\alpha \kappa n(n-1)} & \text { if } \bar{n}(\kappa) \leq n<\overline{\bar{n}}(\kappa) \\ 1 & \text { if } n \geq \overline{\bar{n}}(\kappa)\end{cases}
$$

where

$$
\bar{n}(\kappa)=\frac{\psi t+\alpha}{2 \alpha}+\sqrt{\left(\frac{\psi t+\alpha}{2 \alpha}\right)^{2}-\frac{\psi t}{\alpha \kappa}}
$$

and

$$
\overline{\bar{n}}(\kappa)=\frac{\psi t+\alpha(1-\psi)}{2 \alpha(1-\psi)}+\sqrt{\left(\frac{\psi t+\alpha(1-\psi)}{2 \alpha(1-\psi)}\right)^{2}-\frac{\psi t}{\alpha \kappa(1-\psi)}} .
$$

As $\bar{n}=\bar{n}(1)$ and $\overline{\bar{n}}=\overline{\bar{n}}(1)$, we can state our second main result.

\footnotetext{
${ }^{20}$ For instance, in 2010 Clear Channel owned over 1200 radio stations, some of which in direct competition with each other, Columbia Jounalism Review, Who Owns What, 2010.
} 


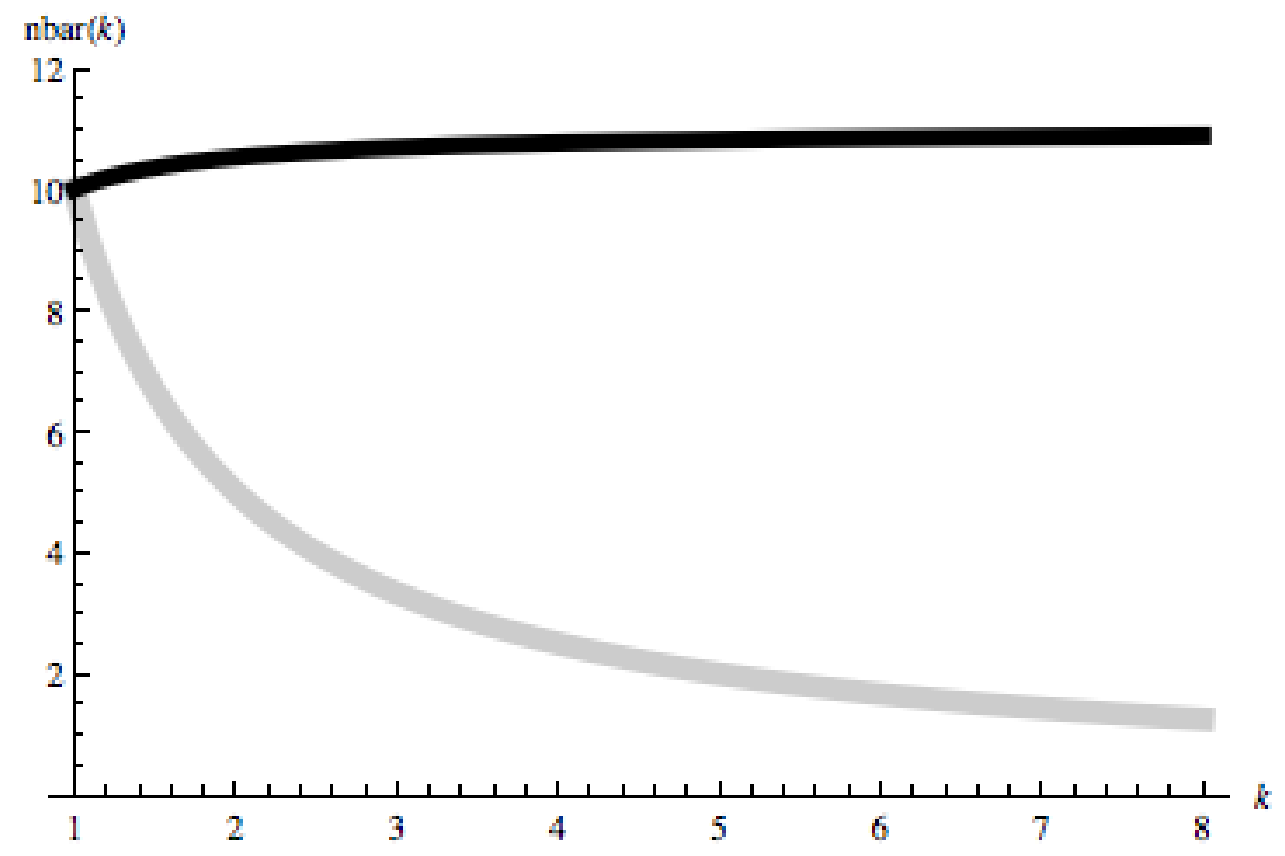

Figure 2: $\bar{n}(\kappa)$ [black] and $\frac{\bar{n}}{\kappa}$ [grey] as a function of $\kappa[t=5 ; \alpha=\psi=.1]$

Proposition 4 In a market with $n \geq 2$ media firms, each one of which owns $\kappa \geq 1$ purely advertising funded media outlets, there is a unique symmetric equilibrium with: severe bias $\left(x_{k_{i}}=0\right.$ for all $i$ and $\left.k_{i}\right)$ when $n<\bar{n}(\kappa)$; no bias $\left(x_{k_{i}}=1\right.$ for all $i$ and $k_{i}$ ) when $n>\overline{\bar{n}}(\kappa)$; and with intermediate bias $\left(x_{k_{i}}=\frac{1}{\psi}-\frac{(\kappa n-1) t}{\alpha \kappa n(n-1)} \in[0,1]\right.$ for all $i$ and $\left.k_{i}\right)$ when $\bar{n}(\kappa) \leq n \leq \overline{\bar{n}}(\kappa)$.

Moreover, allowing firms to own multiple media outlets makes self-censorship more likely in the the following sense,

$$
\bar{n}(\kappa) \geq \bar{n} \text { and } \overline{\bar{n}}(\kappa) \geq \overline{\bar{n}}
$$

for all $\kappa \geq 1$, both with strict inequality unless $\kappa=1$, and also

$$
\lim _{\kappa \rightarrow \infty} \bar{n}(\kappa)=\bar{n}+1 \text { and } \frac{\partial \bar{n}(\kappa)}{\partial \kappa}>0,
$$

and the same holds for $\overline{\bar{n}}(\kappa)$ compared to $\overline{\bar{n}}$.

As expected, what is determinant of whether or not there is self-censorship, and how much of it there is, is not so much the number of media outlets 
$(n \kappa)$ but rather the number of separate owners in the market $(n)$. Somewhat surprisingly, the result is actually stronger. It says that increasing the number of media outlets that any media firm can own in fact aggravates the selfcensorship in the following sense. For a fixed number of owners, the more subsidiary outlets there are, the more likely it is that self-censorship occurs.

In Figure 2, we plot (in black) the number of owners $(\bar{n}(\kappa))$ that are necessary to avoid severe bias $(x=0)$. For comparison we also plot (in grey) $\frac{\bar{n}}{\kappa}$, which is the benchmark number of firms necessary to avoid severe bias if what mattered were only the total number of outlets. ${ }^{21}$ Notice that $\bar{n}(\kappa)$ is not only less steeply downward sloping (than the benchmark $\frac{\bar{n}}{\kappa}$ ), but it is actually upwards sloping! A similar picture obtains for $\overline{\bar{n}}(\kappa)$.

The intuition for this surprising result stems essentially from the assumption that viewers are interested in two outlets only so that allowing firms to own multiple outlets gives them additional local monopoly power. Notice that with $n$ owners each of which owns $\kappa$ media outlets, the share of consumers that are "trapped" between two outlets of any of the single owners is $\frac{\kappa-1}{\kappa n-1}\left(=n \frac{\kappa(\kappa-1)}{2} \frac{2}{\kappa n(\kappa n-1)}\right)$, which goes from 0 to $\frac{1}{n^{2}}$ as $\kappa$ goes from 1 to $\infty$. The phenomenon of having some share of the audience captured by the same owner is not totally unrealistic in practice.

At the same time, it should be stressed that the result also clearly depends on the fact that transportation costs are assumed to be constant as one varies the number of outlets. With transportation costs decreasing with the number of outlets added to the network, the numbers $\bar{n}(\kappa)$ and $\overline{\bar{n}}(\kappa)$ might also decrease depending on how fast transportation costs are decreasing. As we discuss in Section 5 below, we believe that the fact that the slope of $\bar{n}(\kappa)$ and $\overline{\bar{n}}(\kappa)$ is less than that of $\frac{\bar{n}}{\kappa}$ to be the robust finding. Nonetheless, the fact that it is actually increasing in $\kappa$ here is worth noting also since the constant transportation cost is an important benchmark which in certain circumstances may also have empirical validity.

\footnotetext{
${ }^{21}$ For an alternative derivation of $\frac{\bar{n}}{\kappa}$, assume the owners sub-optimally maximized the profit of each outlet individually, without taking into account their power to simultaneously change the behavior of all their outlets. Then the number of outlets necessary to avoid severe bias would be the original $\bar{n}$ from the case $\kappa=1$, but because each owner owns $\kappa$ outlets, the number of owners necessary would be $\frac{\bar{n}}{\kappa}$.
} 
Suppose now firms can also set positive prices $\left(p_{\kappa_{i}} \geq 0\right)$. Again from the FOC's we have

$$
\frac{\partial \pi}{\partial y_{\kappa_{i}}}=\frac{\beta \kappa(n-1)}{\kappa n(\kappa n-1) t}\left(\eta C_{0}+p\right)-\delta y_{\kappa_{i}}
$$

and

$$
\frac{\partial \pi}{\partial p_{\kappa_{i}}}=\frac{1}{\kappa n}-\frac{\kappa(n-1)}{\kappa n(\kappa n-1) t} \eta C_{0}-\frac{\kappa n-1}{\kappa n(\kappa n-1) t} p_{\kappa_{i}}+\sum_{\kappa_{i}^{\prime} \neq \kappa_{i}} \frac{1}{\kappa n(\kappa n-1) t} p_{\kappa_{i}^{\prime}},
$$

which under symmetry implies

$$
p=\left[\frac{(\kappa n-1)}{\kappa(n-1)} t-\eta C_{0}\right]^{+}=0, \quad y=\frac{\beta \kappa(n-1)}{\delta \kappa n(\kappa n-1) t} \eta C_{0},
$$

and which we summarize as follows.

Proposition 5 In a market with $n \geq 2$ media firms, each of which owns $\kappa \geq 1$ media outlets, the level of quality is relatively lower and prices are relatively higher than if there were $\kappa n$ separately owned media firms.

This is intuitive and follows from the decreased competition (and larger "captured" audiences) than in the case of all separately owned firms.

\subsection{The Logit and the Salop models}

We here illustrate the robustness of using the spokes model to describe the oligopolistic interaction between the outlets in deriving the main results of the paper. We consider two well-known yet conceptually quite distinct models of product differentiation, namely the multinomial logit and the Salop (1979) model. $^{22}$ What these models share with the spokes model is that aggregate demand for media is fixed at a mass of unity and does not depend on either the magnitude of the transportation costs (or the degree of substitutability between products) nor on the number of outlets (or products) in the market.

We start with the first main result, namely the existence of certain cut-off numbers of outlets $(\bar{n}, \overline{\bar{n}})$ below which severe bias occurs (see Propositions 1

\footnotetext{
${ }^{22}$ We refer to Tirole (1988) and Anderson et al. (1992) for textbook treatments of these models, in particular for derivations of the corresponding share equations.
} 
and 2). The result goes through qualitatively with slightly different values for the cut-offs, namely, in the logit model,

$$
\bar{n}_{\text {Logit }}=\frac{\psi t}{\alpha}+1, \quad \overline{\bar{n}}_{\text {Logit }}=\frac{\psi t}{\alpha(1-\psi)}+1
$$

obtained from the unrestricted solution $x_{\text {Logit }}(n)=\frac{1}{\psi}-\frac{t}{\alpha(n-1)}$, and for the Salop model,

$$
\bar{n}_{\text {Salop }}=\sqrt{\frac{\psi t}{\alpha}}, \quad \overline{\bar{n}}_{\text {Salop }}=\sqrt{\frac{\psi t}{\alpha(1-\psi)}},
$$

obtained from the unrestricted solution $x_{\text {Salop }}(n)=\frac{1}{\psi}-\frac{t}{\alpha n^{2}} \cdot{ }^{23}$

In particular, if $\bar{n}$ and $\overline{\bar{n}}$ denote the cut-offs computed for the spokes model (see Proposition 1), we have

$$
\bar{n}_{\text {Logit }}=\bar{n}+1, \quad \bar{n}_{\text {Salop }}=\sqrt{\bar{n}}
$$

and the parallel relations for $\overline{\bar{n}}_{\text {Logit }}$ and $\overline{\bar{n}}_{\text {Salop }}$. This establishes a fairly tight relationship between the three models.

Concerning the second main result, namely the existence of cut-off numbers of firms for given numbers of subsidiary outlets, one can again show that first of all these exist in the two alternative models. To see this we report the unrestricted solutions for $x$ in the two models,

$$
x_{\text {Logit }}(n, \kappa)=\frac{1}{\psi}-\frac{t}{\alpha(n-1)}
$$

and

$$
x_{\text {Salop }}(n, \kappa)=\frac{1}{\psi}-\frac{(\kappa n-1) t}{\alpha(\kappa n)^{2}(n-1)}
$$

However, while they both clearly lie above their respective benchmark $\frac{\bar{n}_{\text {Logit }}(1)}{\kappa}$, $\frac{\bar{n}_{\text {Salop }}(1)}{\kappa}$, they do not increase with the number $\kappa$ of subsidiary outlets, which

${ }^{23}$ The easiest way to derive the cut-offs is from our FOC conditions in the Appendix and the corresponding share equations which can be found in Triole (1988), and which take the forms

$$
s_{i, \text { Logit }}=\frac{e^{u_{i} / t}}{(n-1) e^{u / t}+e^{u_{i} / t}}, \quad s_{i, \text { Salop }}=\frac{1}{n}+\frac{u_{i}-u}{t},
$$

where $u$ is the utility level offered by the other outlets. 
is what happens with the spokes model. In the case of the logit model, we can say more. Namely, the cutoffs do not depend on $\kappa$ (i.e., $\bar{n}_{\text {Logit }}(\kappa)=\bar{n}_{\text {Logit }}(1)$ for all $\kappa)$ ! This means that the number of owners is exactly what matters in determining the likelihood of self-censorship in the logit model. Given the benchmark-like nature of this case, we state it formally.

Proposition 6 In a market with $n \geq 2$ media firms, each one of which owns $\kappa \geq 1$ purely advertising funded media outlets competing within a multinomial logit model, there is a unique symmetric equilibrium where the cut-offs $\bar{n}_{\text {Logit }}$ and $\overline{\bar{n}}_{\text {Logit }}$ do not depend on the number of subsidiary outlets $\kappa$.

In particular, allowing firms to own multiple outlets does not affect the likelihood of self-censorship within the multinomial logit model, and for any $\kappa$ the symmetric equilibrium corresponds to the one of Proposition 1 where $\bar{n}$ and $\overline{\bar{n}}$ are replaced by $\bar{n}_{\text {Logit }}=\bar{n}+1$ and $\overline{\bar{n}}_{\text {Logit }}=\overline{\bar{n}}+1$, which do not depend on $\kappa$.

Comparing again with the spokes model, from Proposition 4, we also have for any $\kappa \geq 1$,

$$
\bar{n}_{\text {Logit }}(\kappa)=\bar{n}_{\text {Logit }}=\lim _{\kappa \rightarrow \infty} \bar{n}(\kappa)=\bar{n}+1,
$$

and the parallel relation holds for $\overline{\bar{n}}_{\text {Logit }}(\kappa)$.

We interpret this as suggesting that the number of separate owners of outlets $n(\kappa)$ (rather than the number of outlets $n \kappa$ ) is what really matters in determining the likelihood of self-censorship. This is particularly clear with the logit model. On the other hand, the finding that adding outlets aggravates the likelihood of self-censorship occurs in the spokes model and, for a rather limited set of parameter values, also in the Salop model. In general, whether adding outlets aggravates or in fact alleviates the self-censorship problem depends very much on further aspects of the market such as the transportation costs and the distribution of the audience among the outlets of given owners. ${ }^{24}$ We believe that the benchmark provided by the spokes model can be relevant in contexts where transportation costs do not go down

\footnotetext{
${ }^{24}$ Germano (2007) studies the "uncovered" case of the spokes model, where among other things, total audience increases as outlets are added. In this case the cut-offs $\bar{n}(\kappa)$ and $\overline{\bar{n}}(\kappa)$ decrease with $\kappa$, albeit, again, substantially slower than $\frac{\bar{n}}{\kappa}$.
} 
significantly when adding outlets to the market and where audience can be "captured" by media firms as discussed in Section 3.4 above.

\subsection{Free entry}

Next we investigate the effects of allowing free entry into the above markets.

We assume a fixed cost $K>0$ of operating a given media outlet and solve for the level of fixed costs $\bar{K}$ that supports fully informative equilibria, that is, that supports the possibility of $n>\bar{n}$ firms in the market in equilibrium that make non-negative profits for $K<\bar{K}$.

In the case of $n$ (separately owned) purely commercial media outlets we get as the lower bound for the fixed costs supporting fully informative equilibria,

$$
\bar{K}=\frac{\eta C_{0}}{2 \bar{n}}\left(1-\frac{\eta \beta^{2} C_{0}}{8 \delta \bar{n} t^{2}}\right),
$$

which using $\bar{n}=\frac{t}{2 \alpha}$ reduces to

$$
\bar{K}=\frac{\alpha \eta C_{0}}{t}\left(1-\frac{\alpha \eta \beta^{2} C_{0}}{4 \delta t^{3}}\right)
$$

If actual fixed costs are substantially above the obtained $\bar{K}$, then this means that fully informative market structures cannot be supported.

\section{Welfare analysis}

Our main reason of concern for the possibility of self-censorship stems from potential externalities that can arise from the consequences of not reporting or misreporting on certain key issues such as anthropogenic climate change. ${ }^{25}$ Therefore when assessing the overall welfare we assume there is an additional externality term in the welfare function which depends on $x$. We write overall welfare, evaluated at the symmetric solutions studied in the paper, as

$$
W(x, y)=\alpha x+\beta y+\eta(1-\psi x) C_{0}-\frac{n \delta y^{2}}{2}+\lambda x,
$$

\footnotetext{
${ }^{25}$ As mentioned, a small $\alpha$ need not mean that consumers do not care about the potential externalities but may simply not be aware of or not care about the corresponding information; see Downs (1957) and Hamilton (2004).
} 
where $\lambda \geq 0$ is the weight given to the externalities.

From the FOC we get $y^{*}=\frac{\beta}{\delta n}$ for the quality variable and

$$
x^{*}= \begin{cases}1 & \text { if } \alpha+\lambda \geq \psi \eta C_{0} \\ 0 & \text { else }\end{cases}
$$

for the information variable, so that depending on the size of the externalities generated it may be optimal to set its level equal to 1 or 0 regardless of the number of media firms in the market.

\section{Robustness}

We here relax or replace several of the assumptions made throughout the paper and discuss the robustness of the main findings.

\subsection{Self-censorship on specific or divided issues}

To model the case where advertisers are affected differently by the variable $\mathbf{x}$, we consider a slight generalization of the function $C(\mathbf{x})$ defined in Eq. (4). Let now firms be indexed by $\nu \in[0,1]$ and suppose firm $\nu$ is affected by a marginal amount $\psi(\nu) \in(-1,1)$, which we now allow to be both positive or negative. Then keeping the same (linear) form of demand we can write

$$
\widehat{C}(\mathbf{x})=\int_{\nu \in[0,1]}\left(1-\psi(\nu) \sum s_{i} x_{i}\right) C_{0} d F(\nu),
$$

where $F(\cdot)$ is a distribution function over the firms. The present paper can then be viewed as exploring the consequences on $\mathbf{x}$ in the case where $\hat{\psi} \equiv \int \psi(\nu) d F(\nu)>0$. All the analysis carried out in the previous sections carries over directly after substituting $\hat{\psi}$ for $\psi$. In particular, the cut-offs corresponding to Proposition 1 now take the form

$$
\bar{n}=\frac{\hat{\psi} t}{\alpha}, \quad \overline{\bar{n}}=\frac{\hat{\psi} t}{\alpha(1-\hat{\psi})} .
$$

More specifically, if there is a portion of demand $\left(\nu_{1}\right)$ affected negatively by the variable $\mathbf{x}$, another portion $\left(\nu_{2}\right)$ affected positively, and a remaining 
component that is unaffected. This would give rise to a demand of the form

$$
\begin{aligned}
\widehat{C}(\mathbf{x}) & =\nu_{1}\left(1-\psi_{1} \sum s_{i} x_{i}\right) C_{0}+\nu_{2}\left(1+\psi_{2} \sum s_{i} x_{i}\right) C_{0}+\left(1-\nu_{1}-\nu_{2}\right) C_{0} \\
& =\left(1-\left(\nu_{1} \psi_{1}-\nu_{2} \psi_{2}\right) \sum s_{i} x_{i}\right) C_{0}
\end{aligned}
$$

where $\psi_{1}, \psi_{2}>0$ and $\nu_{1}, \nu_{2} \geq 0, \nu_{1}+\nu_{2}<1$. Since now $\hat{\psi}=\nu_{1} \psi_{1}-\nu_{2} \psi_{2}$, it is easy to see that the more the negative and positive effects balance each other out $\left(\nu_{1} \psi_{1} \approx \nu_{2} \psi_{2}\right)$ the smaller the cut-offs and the less the problem of self-censorship. Similarly, if there are only firms affected negatively but their portion is small $\left(\nu_{2}=0\right.$ and $\left.\nu_{1} \approx 0\right)$ the cut-offs are scaled accordingly.

\subsection{Costly information gathering}

So far throughout the paper, in order to keep the self-censorship separate from considerations of costs of obtaining the information, we assumed that media outlets could access it for free. However, fom the derivation of the optimal levels of quality $\left(y_{i}\right)$, which decrease in the number of outlets, one might think that introducing a cost to access or gather senstive information (say $\frac{v}{2} x_{i}^{2}$ ) might undo the positive effect of competition on the equilibrium level $x_{i}$. This is not the case.

Introducing a cost term to outlet $i$ 's profit function, say $-\frac{v}{2} x_{i}^{2}$, where $v>0$, allows to capture the cost of producing $x_{i}$ in parallel to the cost of producing quality $y_{i}$. Solving the FOC for unrestricted $x \in \mathbb{R}$ gives

$$
x(n)=\frac{\alpha n-\psi t}{v n+\alpha \psi n},
$$

with derivative $\frac{\psi t}{(v+\alpha \psi) n^{2}}>0$ with respect to $n$. Furthermore, this leads to similar results concerning the cut-offs $\bar{n}$ and $\overline{\bar{n}}$ of Proposition 1, namely $\bar{n}_{v}=\frac{\psi t}{\alpha}=\bar{n}$ while $\overline{\bar{n}}_{v}>\overline{\bar{n}}$, for any $v>0$ (in fact, for $v>0$, it could happen that $x<1$ for all $n$ no matter how large). Figure 3 sketches equilibrium accuracy for the cases $v=0$ (in grey) and $v=.1$ (in black).

This is particularly interesting since it suggests not only that our previous conclusions are robust, but moreover it points to the sensitivity of self-censorship to the costs in gathering news. Introducing even slight costs 


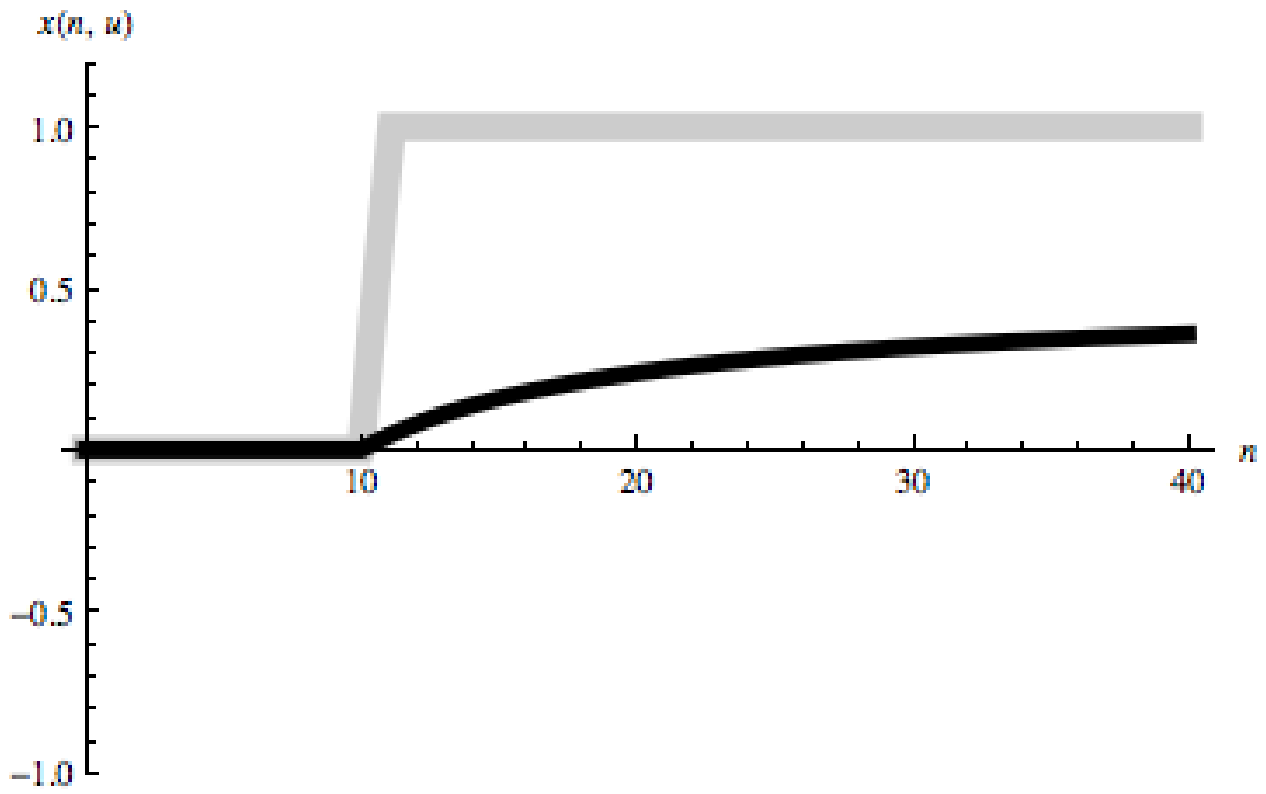

Figure 3: $x(n)$ as a function of $n$ with $v=0$ [grey] and with $v=.1$ [black]

of gathering sensitive information can further dramatically increase the selfcensorship problem. In times where newspapers are under financial constraints and the availability of "public relations" materials relatively (and increasingly) cheap and abundant, it can be particularly attractive for newsrooms to increasingly rely on public relations sources. ${ }^{26}$

\subsection{Information spreading across consumers}

Implicit in our assumption of the aggregate demand for advertisers' products $C(\mathbf{x})$ defined in Eq. (4), it was assumed that each media outlet's coverage can only affect its own audience share. One might wonder what happens if there are spillovers across outlets. To study this case consider the extreme case

\footnotetext{
${ }^{26}$ A recent PEJ study of $1 / 11 / 2010$, "How News Happens," shows how in the Baltimore area, in the third week of July 2009, around eight out of ten stories reported "simply repeated or repackaged previously published information"; also $95 \%$ of the stories that contained new information originated from traditional media (most of them from newspapers); and of the new stories over $80 \%$ originated directly from either government sources or other interest groups. See also Davies (2008) and McChesney and Nichols (2010) on the increasing importance of public relations industries.
} 
where whatever is reported by any one of the outlets immediately reaches all viewers. The demand function in this case would take the form

$$
\widetilde{C}(\mathbf{x})=\left(1-\psi \cdot \max \left\{x_{1}, \cdots, x_{n}\right\}\right) \cdot C_{0} .
$$

In this case, it is easy to see that there will always be an equilibrium with full accuracy for any number of firms in the market. However, there is also an equilibrium with self-censorship whenever the parameters are such that $\bar{n}>1$ in the benchmark model. To see this consider the FOC, now assuming $x_{j}=0$ for all $j \neq i$,

$$
\frac{\partial \pi_{i}}{\partial x_{i}}=\eta\left(\frac{\partial s_{i}}{\partial x_{i}} \widetilde{C}+s_{i} \frac{\partial \widetilde{C}}{\partial x_{i}}\right)=\frac{\eta C_{0}}{n t}\left(\alpha\left(1-\psi x_{i}\right)-\psi t\right)
$$

since now $\frac{\partial \widetilde{C}}{\partial x_{i}}=-\eta \psi$. In particular $i$ 's (unrestricted) best response is to choose $x_{i}=\frac{1}{\psi}-\frac{t}{\alpha}$, where

$$
x_{i} \leq 0 \Longleftrightarrow \frac{\psi t}{\alpha} \geq 1 \quad \text { and } \quad x_{i} \geq 1 \Longleftrightarrow \frac{\psi t}{\alpha(1-\psi)} \leq 1
$$

Note that in the benchmark model $\bar{n}=\frac{\psi t}{\alpha}$ and $\overline{\bar{n}}=\frac{\psi t}{\alpha(1-\psi)}$. This means that, if the parameters of the model are such that $\bar{n}<1$, then self-censorship is not a best-reply for $i$ and so is not an equilibrium if information spreads immediately across consumers, but it is also not an equilibrium in the benchmark model. If, however, the parameters are such that $\bar{n} \geq 1$ then self-censorship is an equilibrium for any $n$ in the model where information spreads whereas it is an equilibrium in the benchmark model only if $n \leq \bar{n}$. In other words, allowing inormation to spread across outlets allows for equilibria with more self-censorship, meaning that (in the extreme case of perfect spreading across outlets) self-censorship can occur whenever it would occur in the benchmark model with a single outlet, but it would now occur regardless of the number of outlets in the market.

\subsection{Individual advertising contracts}

In this paper we have focused on advertising payments determined by the advertisers' total sales and by the audience shares of the outlets; in particular, 


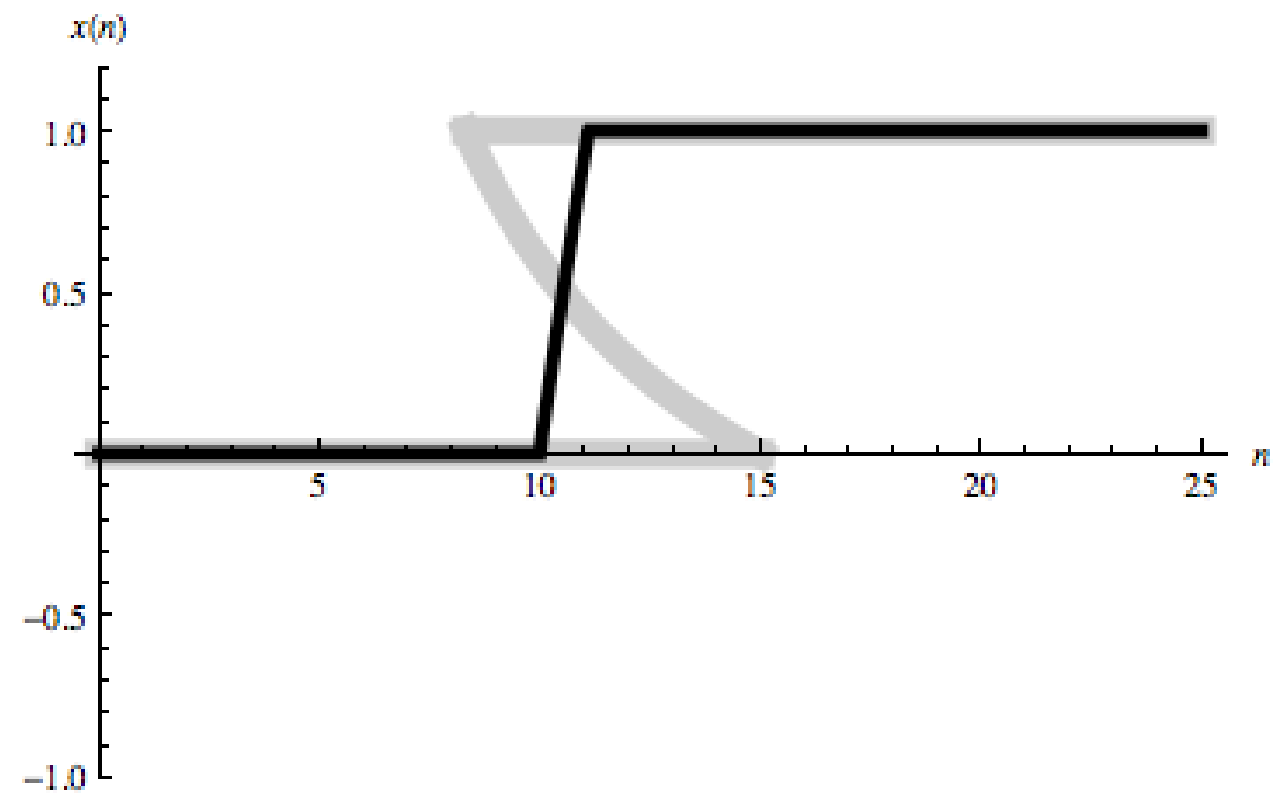

Figure 4: $x(n)$ as a function of $n$ with $\alpha$ fixed [black] and with $\alpha(x)$ [grey]

they were not made contingent upon a certain type of coverage by the outlets. Often, however, advertisers do make personalized contracts which allow for the possibility of withdrawing its advertising when observing a media outlet reporting too heavily on sensitive issues. This was modeled explicitly in Ellman and Germano (2009, Section 4) and can be captured in our model, e.g., by multiplying firm $i$ 's advertising revenues by $\nu_{i}\left(x_{i}\right)=\mathbf{1}_{\left\{x_{i} \leq \bar{x}\right\}}$, meaning that advertisers immediately withdraw their ads whenever $x_{i}>\bar{x}$ and $\bar{x} \in[0,1)$. This strengthens our case for self-censorship to the extent that it increases incentives for media outlets to set $x_{i} \leq \bar{x}$ without weakening incentives to otherwise set $x_{i}=0$ coming from the analysis of Section 3 and also without weakening the effect of concentration on the choice of $x_{i}$.

\section{5 (Un)awareness and acquired tastes}

An aspect of the media that is often considered important is the endogeneity of tastes and awareness of the consumers. Here this could be modeled as allowing the parameter $\alpha$ depend on the level of reporting on sensitive issues 
so that $\alpha(\mathbf{x})$ is weakly increasing in $x$. The idea is that low levels of reporting on an issue generate less awareness and therefore also less "taste" for the issue, or alternatively, repeated reporting on an issue can increase awareness and taste for it. If one adds this feature to the model, then, depending on the strength of the reinforcement effect, one can obtain a tendency towards multiple equilibria in the sense that, everithing else equal, there can be both more equilibria with severe bias for a larger numbers $n>\bar{n}$ and at the same time more equilibria with no bias for smaller numbers $n<\overline{\bar{n}}$. Without going into formal derivations, Figure 4 sketches the larger (grey) set of equilibria. ${ }^{27}$

\section{Concluding remarks}

At a time when journalism and news media are facing serious financial difficulties, it may seem natural to allow and maybe even encourage mergers between media firms. This paper recommends caution with such measures and emphatically argues against it in markets where the ownership structure is already more concentrated than suggested by the cut-offs $(\bar{n}$ and $\overline{\bar{n}})$. Mergers in such concentrated markets would strengthen self-censorship and therefore lead further away from the democratic ideal of an informed public.

To take a last example. Consider the recent - and not so recent - coverage of the health care reform in the US. ${ }^{28}$ With an expenditure of around $17 \%$ of GDP (or over $\$ 7.200$ per capita per year), health care in the US is about twice as expensive per capita as in other developed countries. At the same time, with over 45 million uninsured it consistently ranks at the lower end of studies evaluating health care systems in industrialized countries. ${ }^{29}$ The efforts of the current administration to pass legislation to reform the health care system fell short of solving key issues like providing universal health care coverage

\footnotetext{
${ }^{27}$ The reason the locus of unrestricted $(x, n)$ 's can now be (partially) downward sloping is due not to the introduced non-linearites but to non-convexities of preferences.

${ }^{28}$ See e.g., B. Ehrenreich, "The Medical-Industrial Complex," New Yorker Review of Books, December 17, 1970, for a view of 40 years ago.

${ }^{29}$ E.g., the recent Commonwealth Fund study ranks the US system last among a group of industrialized countries including Germany, The Netherlands, United Kingdom, New Zealand and Canada, reported in Bloomberg Businessweek, "US Health care ranks low among developed nations," June 23, 2010.
} 
and a public health plan option, ${ }^{30}$ that would have moved the system closer to some of the "better" performing ones in Europe and elsewhere. As some argue, these issues were never really taken seriously by mainstream media. ${ }^{31}$ For us, this is a further example of a topic with important externalities and substantial commercial interests that might potentially be subject to commercial bias. $^{32}$

As with the our previous examples, externalities from potentially commercially biased coverage can be large; though, clearly there are many other channels that can lead to biased coverage in the media and many more that can impede "reasonable" policies from being adopted. Already Adam Smith (1904, I.11.264) remarked that

"profit" earners "employ the largest capitals, and by their wealth draw to themselves the greatest share of the public consideration;"

and at the same time also pointed to the fact that their interests and those of the rest of society could be in conflict with each other. To the extent that the media play an decisive role in the political decision-making process, Smith's remarks get to the essence of some our concerns, namely, that advertiser funded media may fail to provide adequate coverage on certain issues of concern to society.

Arguing from a different angle, Downs (1957) (see also Hamilton, 2004)

\footnotetext{
${ }^{30}$ Or more fundamentally of changing "how medical care is organized, paid for and delivered;" see A. Relman, "Health Care: The Disquieting Truth," New Yorker Review of Books, September 30, 2010. Relman also points to the "commercialization" or "profitdriven" aspects of large segments of the US health system as a main source of higher costs of health care in the US; see also A. Relman, "The Health Reform We Need and Are Not Getting," New Yorker Review of Books, July 2, 2009.

${ }^{31}$ E.g., CNN senior medical corespondent Elizabeth Cohen stated: "Fifteen years ago you [heard] people saying: Let's have a single-payer system like Canada [..] You don't hear that as much as you used to. So people are on the same page more than they once were." (CNN, March 5, 2009). See also the mentioned Pew reports of 8/6/2009 and 3/23/2010 on the public's discontent with the general media coverage of the health care reform.

${ }^{32}$ See e.g., M. Tomasky, "The Money Fighting Health-Care Reform," New Yorker Review of Books, April 8, 2010, or Eaton, J., Pell, M.B., and A. Mehta, "Washington Lobbying Giants Cash in on Health Reform Debate," Center for Public Integrity, March 26, 2010, on the extent of commercial interests involved in the health care reform; many of the private companies involved (insurance, pharmaceutical, medical service providers) clearly also had common interests on many key issues at stake.
} 
argued that news relevant to individuals as citizens and to their political choices would be underprovided due to free-riding and insufficient ("rational") demand on their part. In our model, such low demand can be captured by a small preference parameter $(\alpha \approx 0)$, making such topics particularly prone to underreporting in equilibrium (see the formulas for the cut-offs $\bar{n}$ and $\overline{\bar{n}}$ ). In other words, the sensitivity to advertisers combined with this additional effect of being in low demand by viewers, suggests topics particularly vulnerable to self-censorship.

Clearly, more empirical work is needed to validate the picture sketched by the present paper, particularly the phenomenon and extent of advetiser driven bias, identifying relevant topics and the role of concentration in media markets, as well as that of the number of subsidiary outlets owned. The formulas and stylized facts derived can all in principle be tested emprically. Besides measures of media bias, ${ }^{33}$ the formulas depend on measures of concentration, transportation costs, ${ }^{34}$ interest in (or awareness of) sensitive topics, ${ }^{35}$ advertising budgets, and the potential effect on sales of reporting on the given topics.

Gentzkow and Shapiro (2010) study political bias in US daily newspapers. Using sophisticated empirical methodology, they conclude that readers' own ideological biases are a significant factor driving political slant; they find much less evidence of slant originating from media owners' ideological biases. Their paper does not, however, touch on the issue of commercial bias. It would be instructive to apply their methodology to test for this. Separating advertiser from demand driven bias may prove difficult, and it may be necessary to endogenize viewers' demand to allow for actual coverage and positions taken by political parties to influence it. Analyzing the coverage of the health care reform in the US or of climate change (ideally cross-country) would be fascinating and hopefully not impossible.

\footnotetext{
${ }^{33}$ E.g., Boykoff and Boykoff (2004), Reuter and Zitzweitz (2006), or Gentzkow and Shapiro (2010).

${ }^{34}$ Bronnenberg et al. (2010) can be interpreted as estimating empirical transportation costs for consumer packaged goods.

${ }^{35}$ The PEJ and the Pew Research Center keep respectively a News Coverage Index and a News Interest Index for what are most covered and most followed stories on a weekly basis in US media.
} 


\section{Appendix}

Proofs of Propositions 1-3. We need to show existence and uniqueness of the symmetric equilibrium characterized in the propositions. We show it directly for the audience and advertising funded case. Compute

$$
\begin{gathered}
\frac{\partial s_{i}}{\partial x_{i}}=\frac{\alpha}{n t}, \quad \frac{\partial s_{i}}{\partial y_{i}}=\frac{\beta}{n t}, \quad \frac{\partial s_{i}}{\partial p_{i}}=-\frac{1}{n t} \\
\frac{\partial s_{i}}{\partial x_{j}}=-\frac{\alpha}{n(n-1) t}, \quad \frac{\partial s_{i}}{\partial y_{j}}=-\frac{\beta}{n(n-1) t}, \quad \frac{\partial s_{i}}{\partial p_{j}}=\frac{1}{n(n-1) t} \\
\frac{\partial C}{\partial x_{i}}=(-\psi) C_{0}\left(s_{i}+\frac{\partial s_{i}}{\partial x_{i}} x_{i}+\sum_{j \neq i} \frac{\partial s_{j}}{\partial x_{i}} x_{j}\right) \\
=(-\psi) C_{0}\left(s_{i}+\frac{\alpha}{n t} x_{i}-\sum_{j \neq i} \frac{\alpha}{n(n-1) t} x_{j}\right) .
\end{gathered}
$$

Assuming symmetry we have $x_{i}=x, y_{i}=y, p_{i}=p$ and $s_{i}=\frac{1}{n}$ for all $i$, so that

$$
\frac{\partial C}{\partial x_{i}}=-\psi C_{0}\left(\frac{1}{n}+0\right)=\frac{-\psi C_{0}}{n}
$$

The fact that $\frac{\partial C}{\partial x_{i}}$ is negative and decreasing in $n$ in absolute value is crucial for the main results of the paper.

Marginal profits under symmetry are given by

$$
\begin{aligned}
\frac{\partial \pi_{i}}{\partial x_{i}} & =\eta\left(\frac{\partial s_{i}}{\partial x_{i}} C+s_{i} \frac{\partial C}{\partial x_{i}}\right)+p_{i} \frac{\partial s_{i}}{\partial x_{i}} \\
& =\eta\left(\frac{\alpha}{n t}(1-\psi x) C_{0}+\frac{1}{n} \frac{\left(-\psi C_{0}\right)}{n}\right)+p \frac{\alpha}{n t} \\
& =\frac{\eta C_{0}}{n t}\left(\alpha(1-\psi x)-\frac{\psi t}{n}\right)+p \frac{\alpha}{n t} \\
\frac{\partial \pi_{i}}{\partial y_{i}} & =\eta\left(\frac{\partial s_{i}}{\partial y_{i}} C+s_{i} \frac{\partial C}{\partial y_{i}}\right)+p_{i} \frac{\partial s_{i}}{\partial y_{i}}-\delta y_{i} \\
& =\eta\left(\frac{\beta}{n t}(1-\psi x) C_{0}\right)+p \frac{\beta}{n t}-\delta y
\end{aligned}
$$




$$
\begin{aligned}
\frac{\partial \pi_{i}}{\partial p_{i}} & =\eta\left(\frac{\partial s_{i}}{\partial p_{i}} C+s_{i} \frac{\partial C}{\partial p_{i}}\right)+\left(s_{i}+p_{i} \frac{\partial s_{i}}{\partial p_{i}}\right) \\
& =\eta\left(\frac{(-1)}{n t}(1-\psi x) C_{0}\right)+\left(\frac{1}{n}+p \frac{(-1)}{n t}\right)
\end{aligned}
$$

Solved for (unrestricted) $(x, y, p)$ this yields,

$$
p=t-\eta C, y=\frac{\beta \eta C}{\delta n t} \text { and } x=\frac{1}{\psi}-\frac{t}{\alpha n} .
$$

To conclude the proof, we need to verify the SOC's. First notice, that all second-order derivatives of the shares $s_{i}, s_{j}$ with respect to $x_{i}, x_{j}$ and also $y_{i}, y_{j}, p_{i}, p_{j}$ for all $i, j$ are all zero. This implies

$$
\frac{\partial^{2} C}{\partial x_{i}^{2}}=(-\psi) C_{0}\left(\frac{\partial s_{i}}{\partial x_{i}}+\frac{\partial s_{i}}{\partial x_{i}}+0\right)=\frac{-2 \alpha \psi C_{0}}{n t}<0 .
$$

Furthermore,

$$
\begin{gathered}
\frac{\partial^{2} \pi_{i}}{\partial x_{i}^{2}}=\eta\left(2 \frac{\partial s_{i}}{\partial x_{i}} \frac{\partial C}{\partial x_{i}}+s_{i} \frac{\partial^{2} C}{\partial x_{i}^{2}}\right)=\frac{-4 \alpha \psi \eta C_{0}}{n^{2} t}, \quad \frac{\partial^{2} \pi_{i}}{\partial y_{i}^{2}}=-\delta, \quad \frac{\partial^{2} \pi_{i}}{\partial p_{i}^{2}}=\frac{-2}{n t} \\
\frac{\partial^{2} \pi_{i}}{\partial x_{i} \partial y_{i}}=\eta \frac{\partial s_{i}}{\partial y_{i}} \frac{\partial C}{\partial x_{i}}=\frac{-\beta \psi \eta C_{0}}{n^{2} t}=\frac{\partial^{2} \pi_{i}}{\partial y_{i} \partial x_{i}} \\
\frac{\partial^{2} \pi_{i}}{\partial x_{i} \partial p_{i}}=\eta \frac{\partial s_{i}}{\partial p_{i}} \frac{\partial C}{\partial x_{i}}+\frac{\partial s_{i}}{\partial x_{i}}=\frac{\psi \eta C_{0}+\alpha n}{n^{2} t}, \quad \frac{\partial^{2} \pi_{i}}{\partial y_{i} \partial p_{i}}=\frac{\beta}{n t}
\end{gathered}
$$

From here, Proposition 1 follows immediately. Propositions 2 and 3 follow after checking negative definiteness of the matrix of second derivatives $H$ on the relevant subspaces, which follows from checking positive definiteness for the following matrix

$$
P=-H=\left(\begin{array}{ccc}
\frac{4 \alpha \psi \eta C_{0}}{n^{2} t} & \frac{\beta \psi \eta C_{0}}{n^{2} t} & -\frac{\psi \eta C_{0}+\alpha n}{n^{2} t} \\
\frac{\beta \psi \eta C_{0}}{n^{2} t} & \delta & -\frac{\beta}{n t} \\
-\frac{\psi \eta C_{0}+\alpha n}{n^{2} t} & -\frac{\beta}{n t} & \frac{2}{n t}
\end{array}\right)
$$

also on the relevant subspaces. In particular, we can use $t \leq \eta C_{0}, \frac{1}{\psi}-\frac{t}{\alpha n} \leq 1$, and $n \leq \frac{\psi t}{\alpha(1-\psi)}$, since outside these bounds either $x_{i}$ or $p_{i}$ is fixed and so the relevant submatrix is positive definite. It suffices therefore to check that (1) 
$\operatorname{det} P_{1}>0,(2) \operatorname{det} P_{2}>0$, and (3) $\operatorname{det} P>0$, where $P_{1}$ and $P_{2}$ are respectively the top-left $1 \times 1$ and $2 \times 2$ submatrices of $P$. Clearly, (1) is satisfied; (2) reduces to $4 \alpha \delta n^{2} t>\beta^{2} \psi \eta C_{0}$ which with $\delta \geq \frac{\eta C_{0}}{n}$ and the conditions above reduces to $\beta<2 t$; (3) reduces to

$$
t\left(4 \alpha n \psi \eta C_{0}-\left(\alpha n-\psi \eta C_{0}\right)^{2}\right) \geq 2 \alpha n \psi \beta^{2},
$$

which again using the above conditions holds whenever $\beta \leq t$. The latter condition is assumed throughout the paper.

Proof of Proposition 4. For $j \neq \kappa_{i}$ we have

$$
\begin{gathered}
\frac{\partial s_{\kappa_{i}}}{\partial x_{\kappa_{i}}}=\frac{\alpha}{\kappa n t}, \quad \frac{\partial s_{\kappa_{i}}}{\partial y_{\kappa_{i}}}=\frac{\beta}{\kappa n t}, \quad \frac{\partial s_{\kappa_{i}}}{\partial p_{\kappa_{i}}}=-\frac{1}{\kappa n t} \\
\frac{\partial s_{j}}{\partial x_{\kappa_{i}}}=-\frac{\alpha}{\kappa n(\kappa n-1) t}, \quad \frac{\partial s_{j}}{\partial y_{\kappa_{i}}}=-\frac{\beta}{\kappa n(\kappa n-1) t}, \quad \frac{\partial s_{j}}{\partial p_{\kappa_{i}}}=\frac{1}{\kappa n(\kappa n-1) t} \\
\frac{\partial C}{\partial x_{\kappa_{i}}}=\left(s_{\kappa_{i}}+\frac{\partial s_{\kappa_{i}}}{\partial x_{\kappa_{i}}} x_{\kappa_{i}}+\sum_{j \neq \kappa_{i}} \frac{\partial s_{j}}{\partial x_{\kappa_{i}}} x_{j}\right)(-\psi) C_{0} \\
=\left(s_{\kappa_{i}}+\frac{\alpha}{\kappa n t} x_{\kappa_{i}}-\sum_{j \neq \kappa_{i}} \frac{\alpha}{\kappa n(\kappa n-1) t} x_{j}\right)(-\psi) C_{0},
\end{gathered}
$$

which under symmetry $\left(x_{j}=x_{\kappa_{i}}\right)$ yields

$$
\frac{\partial C}{\partial x_{\kappa_{i}}}=\frac{-\psi C_{0}}{\kappa n}
$$

Notice also that $\frac{\partial C}{\partial y_{\kappa_{i}}}=\frac{\partial C}{\partial p_{\kappa_{i}}}=0$. From this we compute the FOC's (again 
using symmetry across both outlets and firms).

$$
\begin{aligned}
\frac{\partial \pi_{i}}{\partial x_{\kappa_{i}}}= & \frac{\partial}{\partial x_{\kappa_{i}}}\left(s_{\kappa_{i}} \eta C-\frac{\delta}{2} y_{\kappa_{i}}^{2}\right)+\sum_{\kappa_{i^{\prime}} \neq \kappa_{i}} \frac{\partial}{\partial x_{\kappa_{i}}}\left(s_{\kappa_{i^{\prime}}} \eta C-\frac{\delta}{2} y_{\kappa_{i^{\prime}}}^{2}\right) \\
= & \eta\left(\frac{\partial s_{\kappa_{i}}}{\partial x_{\kappa_{i}}} C+s_{\kappa_{i}} \frac{\partial C}{\partial x_{\kappa_{i}}}\right)+\sum_{\kappa_{i^{\prime} \neq \kappa_{i}}} \eta\left(\frac{\partial s_{\kappa_{i^{\prime}}}}{\partial x_{\kappa_{i}}} C+s_{\kappa_{i^{\prime}}} \frac{\partial C}{\partial x_{\kappa_{i}}}\right) \\
= & \eta\left(\frac{\alpha}{\kappa n t}(1-\psi x) C_{0}+\frac{1}{\kappa n} \frac{\left(-\psi C_{0}\right)}{\kappa n}\right) \\
& +\sum_{\kappa_{i^{\prime}} \neq \kappa_{i}} \eta\left(\frac{-\alpha(1-\psi x) C_{0}}{\kappa n(\kappa n-1) t}+\frac{1}{\kappa n} \frac{\left(-\psi C_{0}\right)}{\kappa n}\right) \\
= & \frac{\eta C_{0}}{\kappa n}\left(\frac{\alpha \kappa(n-1)(1-\psi x)}{(\kappa n-1) t}-\frac{\psi}{n}\right)
\end{aligned}
$$

Solving for unrestricted $x \in \mathbb{R}$, yields in particular

$$
x(n, \kappa, t)=\frac{1}{\psi}-\frac{(\kappa n-1) t}{\alpha \kappa n(n-1)},
$$

as the locus of (unrestricted and symmetric) $(x, n, \kappa, t)$ 's such that $\frac{\partial \pi}{\partial x_{\kappa_{i}}}=0$. To take into account the constraint $x \in[0,1]$ we need to solve $x(n, \kappa, t)=0$ and $x(n, \kappa, t)=1$ for $n$, which gives the solutions respectively

$$
\bar{n}(\kappa)=\frac{\psi t+\alpha}{2 \alpha}+\sqrt{\left(\frac{\psi t+\alpha}{2 \alpha}\right)^{2}-\frac{\psi t}{\alpha \kappa}}
$$

and

$$
\overline{\bar{n}}(\kappa)=\frac{\psi t+\alpha(1-\psi)}{2 \alpha(1-\psi)}+\sqrt{\left(\frac{\psi t+\alpha(1-\psi)}{2 \alpha(1-\psi)}\right)^{2}-\frac{\psi t}{\alpha \kappa(1-\psi)}},
$$

which are easily verified to satisfy the properties stated in the proposition.

Finally, to check the SOC's notice that

$$
\begin{aligned}
\frac{\partial^{2} \pi_{i}}{\partial x_{\kappa_{i}} \partial x_{\kappa_{i^{\prime}}}}= & \eta\left(\frac{\partial s_{\kappa_{i}}}{\partial x_{\kappa_{i}}} \frac{\partial C}{\partial x_{\kappa_{i^{\prime}}}}+\frac{\partial s_{\kappa_{i}}}{\partial x_{\kappa_{i^{\prime}}}} \frac{\partial C}{\partial x_{\kappa_{i}}}\right)+\eta\left(\frac{\partial s_{\kappa_{i^{\prime}}}}{\partial x_{\kappa_{i}}} \frac{\partial C}{\partial x_{\kappa_{i^{\prime}}}}+\frac{\partial s_{\kappa_{i^{\prime}}}}{\partial x_{\kappa_{i^{\prime}}}} \frac{\partial C}{\partial x_{\kappa_{i}}}\right) \\
& +\sum_{\kappa_{i^{\prime \prime} \neq \kappa_{i}, \kappa_{i^{\prime}}}} \eta\left(\frac{\partial s_{\kappa_{i^{\prime \prime}}}}{\partial x_{\kappa_{i}}} \frac{\partial C}{\partial x_{\kappa_{i^{\prime}}}}+\frac{\partial s_{\kappa_{i^{\prime \prime}}}}{\partial x_{\kappa_{i^{\prime}}}} \frac{\partial C}{\partial x_{\kappa_{i}}}\right),
\end{aligned}
$$


which reduces to

$$
\begin{aligned}
\frac{\partial^{2} \pi_{i}}{\partial x_{\kappa_{i}}^{2}} & =\frac{-2 \alpha \psi \eta C_{0}}{(\kappa n)^{2} t}+\frac{2 \alpha \psi(\kappa-1) \eta C_{0}}{(\kappa n)^{2}(\kappa n-1) t}=\frac{-2 \alpha \psi \kappa(n-1) \eta C_{0}}{(\kappa n)^{2}(\kappa n-1) t} \\
\frac{\partial^{2} \pi_{i}}{\partial x_{\kappa_{i}} \partial x_{\kappa_{i^{\prime}}}} & =\frac{-2 \alpha \psi(\kappa n-2) \eta C_{0}}{(\kappa n)^{2}(\kappa n-1) t}+\frac{2 \alpha \psi(\kappa-2) \eta C_{0}}{(\kappa n)^{2}(\kappa n-1) t}=\frac{-2 \alpha \psi \kappa(n-1) \eta C_{0}}{(\kappa n)^{2}(\kappa n-1) t}
\end{aligned}
$$

from which we can immediately see that the relavant matrix of second order derivatives is negative semi-definite for $\kappa \geq 1$.

Proof to Proposition 5. As before we have, $\frac{\partial C}{\partial y_{\kappa_{i}}}=\frac{\partial C}{\partial p_{\kappa_{i}}}=0$, from which we can compute

$$
\begin{aligned}
\frac{\partial \pi}{\partial y_{\kappa_{i}}} & =\frac{\partial}{\partial y_{\kappa_{i}}}\left(s_{\kappa_{i}} \eta C+s_{\kappa_{i}} p_{\kappa_{i}}-\frac{\delta}{2} y_{\kappa_{i}}^{2}\right)+\sum_{\kappa_{i}^{\prime} \neq \kappa_{i}} \frac{\partial}{\partial y_{\kappa_{i}}}\left(s_{\kappa_{i}^{\prime}} \eta C+s_{\kappa_{i}^{\prime}} p_{\kappa_{i}^{\prime}}-\frac{\delta}{2} y_{\kappa_{i}^{\prime}}^{2}\right) \\
& =\left(\frac{\partial s_{\kappa_{i}}}{\partial y_{\kappa_{i}}}\left(\eta C+p_{\kappa_{i}}\right)+\eta s_{\kappa_{i}} \frac{\partial C}{\partial y_{\kappa_{i}}}-\delta y_{\kappa_{i}}\right)+\sum_{\kappa_{i}^{\prime} \neq \kappa_{i}}\left(\frac{\partial s_{\kappa_{i}^{\prime}}}{\partial y_{\kappa_{i}}}\left(\eta C+p_{\kappa_{i}^{\prime}}\right)+\eta s_{\kappa_{i}^{\prime}} \frac{\partial C}{\partial y_{\kappa_{i}}}\right) \\
& =\left(\frac{\beta(\kappa n-1)}{\kappa n(\kappa n-1) t}+\sum_{\kappa_{i}^{\prime} \neq \kappa_{i}} \frac{(-\beta)}{\kappa n(\kappa n-1) t}\right)\left(\eta C_{0}+p\right)-\delta y_{\kappa_{i}} \\
& =\frac{\beta \kappa(n-1)}{\kappa n(\kappa n-1) t}\left(\eta C_{0}+p\right)-\delta y_{\kappa_{i}} \cdot \\
\frac{\partial \pi}{\partial p_{\kappa_{i}}} & =\frac{\partial}{\partial p_{\kappa_{i}}}\left(s_{\kappa_{i}} \eta C+s_{\kappa_{i}} p_{\kappa_{i}}-\frac{\delta}{2} y_{\kappa_{i}}^{2}\right)+\sum_{\kappa_{i}^{\prime} \neq \kappa_{i}} \frac{\partial}{\partial p_{\kappa_{i}}}\left(s_{\kappa_{i}^{\prime}} \eta C+s_{\kappa_{i}^{\prime}} p_{\kappa_{i}^{\prime}}-\frac{\delta}{2} y_{\kappa_{i}^{\prime}}^{2}\right) \\
& =\left(s_{\kappa_{i}}+\frac{\partial s_{\kappa_{i}}}{\partial p_{\kappa_{i}}}\left(\eta C+p_{\kappa_{i}}\right)+\eta s_{\kappa_{i}} \frac{\partial C}{\partial p_{\kappa_{i}}}\right)+\sum_{\kappa_{i}^{\prime} \neq \kappa_{i}}\left(\frac{\partial s_{\kappa_{i}^{\prime}}}{\partial p_{\kappa_{i}}}\left(\eta C+p_{\kappa_{i}^{\prime}}\right)+\eta s_{\kappa_{i}^{\prime}} \frac{\partial C}{\partial p_{\kappa_{i}}}\right) \\
& =\frac{1}{\kappa n}-\frac{\kappa(n-1)}{\kappa n(\kappa n-1) t} \eta C_{0}-\frac{\kappa n-1}{\kappa n(\kappa n-1) t} p_{\kappa_{i}}+\sum_{\kappa_{i}^{\prime} \neq \kappa_{i}} \frac{1}{\kappa n(\kappa n-1) t} p_{\kappa_{i}^{\prime}} \cdot
\end{aligned}
$$

Proof of Proposition 6, the Salop and Logit cases. We only check the case of the multinomial logit model; the case of the Salop model is similar to the other two. Suppose $\kappa \geq 1$, then as before, $\frac{\partial C}{\partial x_{\kappa_{i}}}=\frac{-\psi C_{0}}{\kappa n}$, and, for $j \neq \kappa_{i}$, 
we also compute

$$
\begin{array}{ccc}
\frac{\partial s_{\kappa_{i}}}{\partial x_{\kappa_{i}}}=\frac{\alpha(\kappa n-1)}{(\kappa n)^{2} t}, & \frac{\partial s_{\kappa_{i}}}{\partial y_{\kappa_{i}}}=\frac{\beta(\kappa n-1)}{(\kappa n)^{2} t}, & \frac{\partial s_{\kappa_{i}}}{\partial p_{\kappa_{i}}}=-\frac{\kappa n-1}{(\kappa n)^{2} t} \\
\frac{\partial s_{j}}{\partial x_{\kappa_{i}}}=-\frac{\alpha}{(\kappa n)^{2} t}, & \frac{\partial s_{j}}{\partial y_{\kappa_{i}}}=-\frac{\beta}{(\kappa n)^{2} t}, \quad \frac{\partial s_{j}}{\partial p_{\kappa_{i}}}=\frac{1}{(\kappa n)^{2} t} .
\end{array}
$$

From this we compute the FOC's (again using symmetry across both outlets and firms)

$$
\begin{aligned}
\frac{\partial \pi_{i}}{\partial x_{\kappa_{i}}}= & \eta\left(\frac{\partial s_{\kappa_{i}}}{\partial x_{\kappa_{i}}} C+s_{\kappa_{i}} \frac{\partial C}{\partial x_{\kappa_{i}}}\right)+\sum_{\kappa_{i^{\prime}} \neq \kappa_{i}} \eta\left(\frac{\partial s_{\kappa_{i^{\prime}}}}{\partial x_{\kappa_{i}}} C+s_{\kappa_{i^{\prime}}} \frac{\partial C}{\partial x_{\kappa_{i}}}\right) \\
= & \eta\left(\frac{\alpha(\kappa n-1)}{(\kappa n)^{2} t}(1-\psi x) C_{0}+\frac{1}{\kappa n} \frac{\left(-\psi C_{0}\right)}{\kappa n}\right) \\
& +\sum_{\kappa_{i^{\prime}} \neq \kappa_{i}} \eta\left(\frac{-\alpha}{(\kappa n)^{2} t}(1-\psi x) C_{0}+\frac{1}{\kappa n} \frac{\left(-\psi C_{0}\right)}{\kappa n}\right) \\
= & \frac{\eta C_{0}}{(\kappa n)^{2}}\left(\frac{\alpha((\kappa n-1)-(\kappa-1))(1-\psi x)}{t}-\psi(1+\kappa-1)\right) \\
= & \frac{\eta C_{0}}{\kappa n^{2}}\left(\frac{\alpha(n-1)(1-\psi x)}{t}-\psi\right)
\end{aligned}
$$

Solving for unrestricted $x \in \mathbb{R}$, yields in particular

$$
x(n, \kappa, t)=\frac{1}{\psi}-\frac{t}{\alpha(n-1)},
$$

as the locus of (unrestricted and symmetric) $(x, n, \kappa, t)$ 's such that $\frac{\partial \pi}{\partial x_{\kappa_{i}}}=0$. Notice it does not depend on $\kappa$ ! To take into account the constraint $x \in[0,1]$ we need to solve $x(n, \kappa, t)=0$ and $x(n, \kappa, t)=1$ for $n$, which gives the solutions respectively

$$
\bar{n}(\kappa)=\frac{\psi t}{\alpha}+1 \quad \text { and } \overline{\bar{n}}(\kappa)=\frac{\psi t}{\alpha(1-\psi)}+1,
$$

which are easily verified to satisfy the properties stated in the proposition. Verifying the SOC's, finally, is tedious but analogous to the case of the spokes model. 


\section{References}

[1] Advertising Age (2007) Leading National Advertisers: Marketer Profiles Yearbook, (B. Johnson et al., eds.), New York: The Ad Age Group.

[2] Anderson, S.P., A. de Palma, and J.F. Thisse (1992) Discrete Choice Theory of Product Differentiation, MIT Press, Cambridge, MA.

[3] Anderson, S.P., and J. McLaren (2010) "Media Mergers and Media Bias with Rational Consumers," Mimeo, University of Virginia.

[4] Armstrong, M., and H. Weeds (2007) "Programme Quality in Subscription and Advertising-Funded Television," Mimeo, University College London and University of Essex.

[5] Bagdikian, B.H. (2004) The New Media Monopoly, Beacon Press, Boston

[6] Baghestani, H. (1991) "Cointegration Analysis of the Advertising-Sales Relationship," Journal of Industrial Economics, 39, 671-681.

[7] Baker, C.E. (1994) Advertising and a Democratic Press, Princeton University Press, Princeton.

[8] Baker, C.E. (2007) Media Concentration and Democracy: Why Ownership Matters, Cambridge University Press, Cambridge.

[9] Besley, T., and A. Prat (2006) "Handcuffs for the Grabbing Hand? Media Capture and Government Accountability," American Economic Review, 96, 720-736.

[10] Billett, S. (2010) "Dividing Climate Change: Global Warming in the Indian Mass Media," Climatic Change, 99, 1-16.

[11] Blasco, A., Pin, P., and F. Sobbrio (2010) "Paying Positive to Go Negative: Advertisers' Competition and Media Reports," Mimeo, IMT Lucca.

[12] Boykoff, M.T. (2008) "Lost in Translation? United States Televsion News Coverage of Climate Change," Climatic Change, 86, 1-11. 
[13] Boykoff, M.T., and J.M. Boykoff (2004) "Balance as Bias: Global Warming and the US Prestige Press," Global Environmental Change, 14, 125136.

[14] Bronnenberg, B.J., Dubé, J.-P.H., and M. Gentzkow (2010) "The Evolution of Brand Preferences: Evidence from Consumer Migration," Mimeo, University of Chicago.

[15] Chaloupka, F.J., and K.E. Warner (2000) "The Economics of Smoking," in Handbook of Health Economics, Vl. 1, Edited by A.J. Culyer and J.P. Newhouse, Elsevier Science B.V., 1541-1627.

[16] Chen, Y., and M.H. Riordan (2007) "Price and Variety in the Spokes Model," Economic Journal, 117, 897-921.

[17] Compaine, B.M., and D. Gomery (2000) Who Owns the Media?: Competition and Concentration in the Mass Media Industry, 3rd ed., Lawrence Erlbaum Associates, Mahwah, New Jersey.

[18] Davies, N. (2008) Flat Earth News, Vintage Books, London.

[19] De Smet, D., and S. Vanormelingen (2010), "The Advertiser is Mentioned Twice. Media Bias in Belgian Newspapers," Mimeo, Katholieke Universiteit Leuven.

[20] Downs, A. (1957) An Economic Theory of Democracy, Harper \& Row, New York.

[21] Elliott, C. (2001) "A Cointegration Analysis of Advertising and Sales Data," Review of Industrial Organization, 18: 417-426.

[22] Ellman, M., and F. Germano (2009) "What Do the Papers Sell? A Model of Advertising and Media Bias," Economic Journal, 119, 680704 .

[23] Gal-Or, E., Geylani, T., and T.P. Yildirim (2010) "The Impact of Advertising on Media Bias," Mimeo, University of Pittsburgh. 
[24] Gambaro, M., and R. Puglisi (2009) "What do Ads Buy? Daily Coverage of Listed Companies on the Italian Press," Mimeo, University of Milano and University of Pavia.

[25] Gentzkow, M., and J.M. Shapiro (2006) "Media Bias and Reputation," Journal of Political Economy, 114, 280-316.

[26] Gentzkow, M., and J.M. Shapiro (2008a) "Competition and Truth in the Market for News?," Journal of Economic Perspectives, 22, 133-154.

[27] Gentzkow, M., and J.M. Shapiro (2008b) "Preschool Television Viewing and Adolescent Test Scores: Historical Evidence from the Coleman Study," Quarterly Journal of Economics, 121, 931-972.

[28] Gentzkow, M., and J.M. Shapiro (2010) "What Drives Media Slant? Evidence from U.S. Daily Newspapers," Econometrica, 78, 35-71.

[29] George, L., and J. Waldfogel (2003) "Who Affects Whom in Daily Newspaper Markets?," Journal of Political Economy, 11, 765-785.

[30] Germano, F. (2009) "On Commercial Media Bias," Mimeo, Universitat Pompeu Fabra.

[31] Hamilton, J.T. (2004) All the News That's Fit to Sell: How the Market Transforms Information into News, Princeton University Press, Princeton.

[32] Ho, D.E., and K.M. Quinn (2009) "Viewpoint Diversity and Media Consolidation: An Empirical Study," Stanford Law Review, 61: 781-868.

[33] Jung, C., and B.J. Seldon (1995) "The Macroeconomic Relationship between Advertising and Consumption," Southern Economic Journal, 61: $577-587$.

[34] Lacy, S., and A. Blanchard (2003) "The Impact of Public Ownership, Profits, and Competition on Number of Newsroom Employees and Starting Salaries in Mid-Sized Daily Newspapers," Journalism and Mass Communication Quarterly, 80: 949-968. 
[35] McChesney, R.W. (2004) The Problem of the Media: U.S. Communication Politics in the 21st Century, Monthly Review Press, New York.

[36] McChesney, R.W., and J. Nichols (2010) The Death and Life of American Journalism, Nation Books, Philadelphia.

[37] Mullainathan, S., and A. Shleifer (2005) "The Market for News," American Economic Review, 95, 1031-1053.

[38] Noam, E.M. (2009) Media Ownership and Concentration in America, Oxford University Press, Oxford.

[39] Oreskes, N. (2004) "Beyond the Ivory Tower: The Scientific Consensus on Climate Change," Science, 306, 1686-1686.

[40] Prat, A., and D. Strömberg (2006) "Commercial Television and Voter Information," Mimeo LSE and Stockholm University.

[41] Reuter, J., and E. Zitzewitz (2006) "Do Ads Influence Editors? Advertising and Bias in the Financial Media," Quarterly Journal of Economics, 121, 197-227.

[42] Rinallo, D., and S. Basuroy (2009) "Does Advertising Spending Influence Media Coverage of the Advertiser," Journal of Marketing, 73, 3346.

[43] Schiffrin, A. (2010) Words and Money, Verso, London.

[44] Schmalensee, R. (1972) The Economics of Advertising, North-Holland, Amsterdam.

[45] Smith, A. (1904) An Inquiry into the Nature and Causes of the Wealth of Nations, Methuen \& Co., London.

[46] Steiner, P.O. (1952) "Program Patterns and Preferences, and the Workability of Competition in Radio Broadcasting," Quarterly Journal of Economics, 66, 194-223. 
[47] Strömberg, D. (2004) "Mass Media Competition, Political Competition, and Public Policy," Review of Economic Studies, 71 , 265-284.

[48] Tirole, J. (1988) The Theory of Industrial Organization, MIT Press, Cambridge, MA. 